\title{
On the Free Boundary Problem for Stationary Compressible Navier-Stokes Equations
}

\author{
Konstantin Pileckas ${ }^{1}$ and Wojciech M. Zajaczkowski ${ }^{2}$ \\ ${ }^{1}$ Institute of Mathematics and Cybernetics, AN LITH. SSR, Akademios 4, \\ SU-232001 Vilnus, USSR \\ ${ }^{2}$ Institute of Mathematics, Polish Academy of Sciences, Sniadeckich 8, \\ PL-00-950, Warsaw, Poland
}

\begin{abstract}
We consider the equations which describe a stationary motion of a viscous compressible barotropic fluid in a bounded domain in $\mathbb{R}^{3}$ with a free boundary determined by the surface tension. By means of some a priori estimates we prove the existence of rotationally symmetric solutions (in reality with some additional symmetry) for a sufficiently small external force and in the case of rotationally symmetric force and domain (where also we need more symmetry, respectively).
\end{abstract}

\section{Introduction}

In this paper a free boundary problem for a viscous compressible barotropic fluid is considered. The stationary motion of the fluid in a bounded domain $\Omega \subset \mathbb{R}^{3}$ with a free boundary $S$ is described by the following equations [24]:

$$
\begin{gathered}
\varrho v \nabla v+\nabla p+A v=\varrho f \quad \text { in } \Omega, \\
\operatorname{div}(v \varrho)=0 \quad \text { in } \Omega, \\
v \cdot \bar{n}=0 \quad \text { on } S, \\
T \bar{n}-(\bar{n} T \bar{n}) \bar{n}=0 \quad \text { on } S,
\end{gathered}
$$

where $A v=-\mu \Delta v-v \nabla \operatorname{div} v, v=v(x)$ is the velocity of the fluid, $\varrho=\varrho(x)$ the density $y, p=p(\varrho)$ the pressure (which is a given function of $\varrho$ ), $f=f(x)$ the external force field per unit mass. The viscosity coefficients $\mu$ and $v$ satisfy the thermodynamic restrictions

$$
\mu>0, \quad v \geqq \frac{1}{3} \mu .
$$

We also use the deformation tensor $T$ with elements

$$
T_{i j}=-p \delta_{i j}+T_{i j}^{\prime}(v), \quad i, j=1,2,3,
$$


where

$$
\begin{gathered}
T_{i j}^{\prime}(v)=\mu\left(\frac{\partial v_{i}}{\partial x_{j}}+\frac{\partial v_{j}}{\partial x_{i}}-\frac{2}{3} \delta_{i j} \operatorname{div} v\right)+\xi \delta_{i j} \operatorname{div} v \text { and } \\
v=\xi+\frac{\mu}{3}, \quad \xi \geqq 0
\end{gathered}
$$

By $\bar{n}$ we denote the unit outward normal vector to $S$, and by $\bar{\tau}$ any unit tangent vector.

The unknown free boundary surface $S$ is determined by the equation

$$
\sigma H=\left.\bar{n} T \bar{n}\right|_{S}+p_{0}
$$

where $\sigma$ is the coefficient of surface tension and $H$ is the mean curvature of $S$.

By virtue of $(1.1)_{2,3}$ the total mass of the fluid $\left(M=\int_{\Omega} \varrho\right)$ can be assumed a constant and we denote by $\bar{\varrho}=\frac{M}{|\Omega|}(|\Omega| \equiv \operatorname{vol}(\Omega))$ the mean density of the fluid. So, we prescribe the additional condition

$$
\int_{\Omega} \varrho d x=M
$$

Moreover, in the case of closed surface the following condition must be satisfied (see [11,13] and [19] Chap. 15):

which by Eqs. (1.1) implies

$$
\int_{S} H n d \sigma=0
$$

$$
\int_{\Omega} \varrho f d x=0
$$

In this paper we prove the existence of solutions to a stationary free boundary problem for the motion of a drop of a viscous compressible barotropic fluid. We follow the method of Pukhnachov [34]. Hence the proof is divided into two steps. First, after having found an a priori estimate we prove the existence of solutions to the Navier-Stokes system in a prescribed domain. Next we show the existence of a free surface for given velocity and pressure. Having a priori estimates in Sobolev spaces for solutions of nonlinear Navier-Stokes equations $\left(v \in H^{3}, p \in H^{2}\right)$ a solution of the free boundary equation has to be found in Sobolev-Slobodetskii spaces $\left(H^{7 / 2}\right)$.

Let us describe the first step in more detail. To show the solvability in Sobolev spaces for the compressible stationary Navier-Stokes Eqs. (1.1) in a prescribed domain we use the method of Valli [62]. Therefore we need a priori estimates which are obtained by the method of Valli-Zajaczkowski [63] which however needs some extra consideration because in our case $S \in H^{7 / 2}$. Moreover we must have the solvability of the linear Stokes problem (3.1), (3.2) and of the hyperbolic Eq. (2.13). The sketch of the proof of the regularity of solutions to the Stokes problem (3.1), (3.2) was shown to the authors by Solonnikov. The proof is based on the method of Solonnikov-Shchadilov [47]. Since in [47] the boundary is assumed to be of class $C^{3}$, and we need this result for $S \in H^{7 / 2}$, we repeat the relevant considerations to show 
that $f \in H^{1}, g \in H^{2}, S \in H^{7 / 2}$ imply $v \in H^{3}, p \in H^{2}$ (Theorem 3.2). Moreover, we prove that $S \in H^{5 / 2}, f \in L_{2}, g \in H^{1}$ imply $v \in H^{2}, p \in H^{1}$ (it is interesting to underline that in the case of the Laplace equation $S \in H^{3 / 2}$ does not imply $v \in H^{2}$, but $S \in H^{5 / 2}$ imply $v \in H^{3}$; see the book by Maz'ya-Shaposhnikova [25]). For the reader's convenience we show the existence of solutions to (3.1), (3.2) in the Appendix.

We prove the existence of solutions of our problem by the method of successive approximations where at each step we need the solvability of the Stokes problem (3.1), (3.2). From Solonnikov-Shchadilov [47] we know that in the case when the domain $\Omega$ is obtained by rotation about some vector $B$, the right-hand side of the Stokes system must be orthogonal to $B \times x$. Because at each step of successive approximations we find a solution and a domain it may happen that the domain has rotational symmetry about $B$. Hence to satisfy the condition of solvability to the Stokes problem we assume the symmetry of the domain and of the right-hand side at each step: so in other words the method of successive approximations is constructed in such a way that the necessary symmetry conditions for solving the Stokes problem (3.1), (3.2) are satisfied. That symmetry implies that the solutions also have the same symmetry and the necessary solvability condition is automatically satisfied.

Note that there is another method of solving the stationary Navier-Stokes compressible equations (see H. Beirao da Veiga [7]) but we do not know how to apply that method to our boundary conditions and for $S \in H^{7 / 2}$.

The hyperbolic Eq. (2.13) was considered in the papers of Beirao da Veiga [7], Friedrichs [17], and Valli [62], but our proof of the existence of solutions of (2.13) needs only that $S \in H^{7 / 2}$ (see Lemma 2.6). Therefore for the reader's convenience, using the uniqueness from [7], we give a simple proof of solvability for this equation in $H^{2}$.

Among the three parameters $M$ (total mass), $\bar{\varrho}=\frac{M}{|\Omega|}$ (mean density) and $|\Omega|=\operatorname{vol}(\Omega)$, only one is arbitrary. Assuming that $M$ is arbitrary we get $\Omega$ from (1.6), because $\varrho$ is a solution of the considered problem, and then the mean density is determined.

Finally, we have to underline that the technical condition $\xi \geqq \frac{2}{3} \mu$ [see (4.6)] must be assumed to prove Lemmas 4.1, 4.2.

Now, we make some comments on the literature concerning free boundary problems for the Navier-Stokes system. In the last fifteen years free boundary problems for Navier-Stokes systems have been intensively studied, starting from papers of Pukhnachov [34-37] who considered the stationary incompressible fluid motion. His method (which was later used by other authors [10-16, 26, 29-32, $38-41,45,49-54])$ consists of the following. First, the incompressible fluid motion is considered in a prescribed domain and then a free boundary is found from a surface equation for the given velocity and pressure (these two problems are usually called auxiliary problems). Using the above procedure the solution of the problem is constructed for small data by the method of successive approximations.

A significant part in Pukhnachov's scheme is the investigation of the Stokes system in a prescribed domain. This investigation is based on the theory of general elliptic boundary value problems (see Agmon et al. [1] and Solonnikov [48]) and on the existence and regularity properties for weak solutions of the boundary value 
problem (3.1), (3.2) for the Stokes system, which were proved by Solonnikov and Shchadilov [47].

Stationary flows with a free boundary having no contact with a rigid wall (most convenient for mathematical treatment) were considered by Pukhnachov [34-37], Osmolovskii [28], Ladyzhenskaya and Osmolovskii [22], Bemelmans [10-13], Antonovskii [3, 4], Fujita-Yashima [18]. In [10-13, 22, 28, 34-37] the solvability of these problems was established in Hölder spaces.

In the case when a free boundary contacts a rigid wall the pressure and the derivatives of velocity may have singularities at the contact points. The singularities depend on the contact angle $\Theta$ (in the case $\Theta=\Pi / 2$ they vanish). The problem for $\Theta=\Pi / 2$ was solved by Sattinger [41]. For arbitrary $\Theta \in(0, \Pi)$ such problems were considered by Solonnikov [49-53]. In these papers the solvability of stationary incompressible motions in open containers were investigated. The solvability of the considered problems was proved in weighted Hölder spaces.

Some stationary plane noncompact free boundary problems for an incompressible fluid were considered by Pileckas [29-31], Pileckas and Specovius-Neugebauer [32], Solonnikov [54], and Socolescu [43,44]. The auxiliary problem (solvability of the Stokes and Navier-Stokes systems in a prescribed domain) for a three-dimensional noncompact free boundary problem was considered by Pileckas and Solonnikov [33]. In these papers motions under gravity in open basis where noncompact free boundary surfaces appears were treated. In the above problems weighted Hölder spaces with weights connected with the unboundedness of the domain were used.

Two-fluid stationary free boundary problems were considered by Rivkind [38-40], Bemelmans [10,14], Erunova [15, 16], and Sokolowskii [45]. In [38-40, 11-13] motions of an incompressible drop with a smooth surface in the second fluid or in the air were investigated. The problem of two incompressible fluids in a container was analysed in [15-16]. In this case the surfaces of fluid have contact with walls of the container and at the contact points the solutions may have singularities. In $[15,40]$ the solvability of this problem was established in weighted Sobolev spaces. In [45] a problem on the common motion of two incompressible fluids down an inclined plane was considered. Note that in this case free boundaries of fluids are noncompact.

Several nonstationary free boundary problems for incompressible fluid were considered by Solonnikov [55-60], Beale [5, 6], Allain [2], and Bemelmans [14]. All these papers dealt with evolution of a finite mass of fluid with smooth free boundaries. Teramoto [61] considered a nonstationary flow with noncompact free boundary down an inclined plane.

Nonstationary flows for compressible fluids were considered by Nishida [27].

The authors do not know of any paper dealing with a free boundary problem for the stationary compressible Navier-Stokes equations.

\section{Notation and Auxiliary Results}

Let $\Omega$ be an arbitrary domain in $\mathbb{R}^{n}$ with boundary $\partial \Omega=S$. We denote by $H^{l}(\Omega)$, $l \geqq 0$, the Sobolev-Slobodetskii function space, endowed with the usual norm 
\|\|$_{l, \Omega}$, and by ||$_{p, \Omega}, 1 \leqq p \leqq \infty$, the usual norm in $L_{p}(\Omega)$. Hence, \|\|$_{0, \Omega}=||_{2, \Omega}$. The scalar product in $L_{2}(\Omega)$ is denoted by $(\cdot, \cdot)_{\Omega} \cdot H^{l-1 / 2}(S), l \geqq 1$ an integer, is the space of traces of elements $u \in H^{l}(\Omega)$. The usual norm in $H^{l-1 / 2}(S)$ is denoted by \|\|$_{l-1 / 2, s}$. For $l \geqq 1, l$ an integer, define $H_{0}^{l}(\Omega)=\left\{u \in H^{l}(\Omega): \partial^{k} u / \partial n^{k}=0\right.$ on $S, k=0, \ldots, l-1\}$. The same notation will be used for spaces of vector fields defined in $\Omega$ and on $S$. We shall usually omit the subscript $\Omega$ in the above norms if no confusion can arise. Also, the summation convention will be used.

Writing $\partial \Omega=S \in H^{l-1 / 2}$ will mean that $S$ is locally determined by a function $\varphi \in H^{l-1 / 2}$, i.e. for each $b \in S$ there exists a neighbourhood $U_{b} \subset S$ which in some local coordinates $\{y\}$ is given by the equation $y_{n}=\varphi\left(y^{\prime}\right), y^{\prime}=\left(y_{1}, \ldots, y_{n-1}\right) \in \omega_{d}$ $=\left\{y^{\prime}:\left|y_{i}\right|<d, i=1, \ldots, n-1\right\}$, where $y_{k}$ and $x_{l}$ are connected by the relation

and $\varphi \in H^{l-1 / 2}\left(\omega_{d}\right)$.

$$
y_{k}=\sum_{l=1}^{3} \alpha_{k l}\left(x_{l}-b_{l}\right), \quad \alpha_{3 l}=n_{l}(b)
$$

For vectors $u, v \in H^{1}(\Omega)$ let us introduce

$$
E(u, v)=\int_{\Omega}\left(\frac{\partial u_{i}}{\partial x_{j}}+\frac{\partial u_{j}}{\partial x_{i}}\right)\left(\frac{\partial v_{i}}{\partial x_{j}}+\frac{\partial v_{j}}{\partial x_{i}}\right) d x
$$

We recall that the vectors for which $E(u, u)=0$ form a finite dimensional affine space of vectors such that (see [47])

$$
u=A+B \times x,
$$

where $A, B$ are constant vectors

We define $\tilde{H}(\Omega)=\{u: E(u, u)<\infty, u \cdot \bar{n}=0$ on $S\}$. If $\Omega$ is a region obtained by rotation about a vector $B$ we denote by $H(\Omega)$ the space of functions in $\tilde{H}(\Omega)$ satisfying the condition

$$
\left(u, u_{0}\right)_{\Omega}=0 \text {, }
$$

where $u_{0}=B \times x$; otherwise we set $H(\Omega)=\widetilde{H}(\Omega)$ (see [47]).

Lemma 2.1 [47]. Let $S \in H^{7 / 2}$. Then for each $u \in H(\Omega)$,

where $|\nabla u|^{2}=\frac{\partial u_{i}}{\partial x_{j}} \frac{\partial u_{i}}{\partial x_{j}}$

$$
\|\nabla u\|_{0}^{2} \leqq c E(u, u)
$$

Note that the proof in [47] is for $S \in C^{2}$, so it suffices to use the embedding $H^{7 / 2}(S) \subset C^{2}(S)$.

Lemma 2.2. For each $u \in H(\Omega)$,

$$
\|u\|_{0}^{2} \leqq c E(u, u)
$$

Proof. Using the inequalities

$$
\|u\|_{0, \Omega}^{2} \leqq c\left(\|\nabla u\|_{0, \Omega}^{2}+\|u\|_{0, S}^{2}\right),
$$

(2.4) and (2.7) from [47] gives estimate (2.5). 
Note that Lemmas $2.1,2.2$ imply the equivalence of the norms $\|u\|_{1}$ and $E(u, u)^{1 / 2}$ for $u \in H(\Omega)$.

In a bounded domain $\Omega$ (or in $\mathbb{R}_{+}^{3}$ ), consider the following problem:

$$
\begin{array}{cl}
\operatorname{div} u=g & \text { in } \Omega, \\
u \cdot \bar{n}=0 & \text { on } S
\end{array}
$$

(in the case of $\mathbb{R}_{+}^{3}, S$ is the plane $\left\{x_{3}=0\right\}$ ).

Lemma 2.3. Problem (2.6) has a solution $u \in H(\Omega)$ if one of the following conditions is satisfied:

a) $\Omega=\mathbb{R}_{+}^{3}, g \in L_{2}(\Omega)$.

b) $\Omega$ is a bounded domain with boundary $S \in \operatorname{Lip}, g \in L_{2}(\Omega)$ and

In both cases

$$
(g, 1)_{\Omega}=0
$$

$$
\|u\|_{1} \leqq c\|g\|_{0}
$$

Proof. Let $\varphi$ be the solution of the Neumann problem

$$
\begin{gathered}
\Delta \varphi=g \quad \text { in } \Omega, \\
\partial \varphi / \partial n=0 \quad \text { on } S .
\end{gathered}
$$

Then $u=\nabla \varphi$ satisfies (2.6) and one gets (2.8) from the well-known results for problem (2.9).

Remark 2.1. The more complicated case when $(2.6)_{2}$ is replaced by $\left.u\right|_{S}=0$ was considered in [21], where the solvability and estimate (2.8) were proved for bounded domains $\Omega$ with Lipschitz boundary.

Lemma 2.4. Let $S \in H^{5 / 2}, a \in H^{5 / 2}(S), b \in H^{3 / 2}(S)$. Then these exists a vector field $v \in H^{3}(\Omega)$ such that

and

$$
v \cdot \bar{n}=a, \quad \bar{\tau} T^{\prime}(v) \bar{n}=b \quad \text { on } S
$$

$$
\|v\|_{3, \Omega} \leqq c\left(\|a\|_{5 / 2, S}+\|b\|_{3 / 2, S}\right)
$$

Proof. Note that the boundary conditions (2.10) are invariant with respect to rotation of variables and vectors, i.e. if we use new coordinates $\{y\}$ connected with $\{x\}$ by the relations $y_{k}=\sum_{l=1}^{3} \alpha_{k l} x_{l}, \alpha_{3 l}=n_{l}$, and a new vector $w$ with components $w_{k}=\sum_{l=1}^{3} \alpha_{k l} v_{l}$ we obtain for $w$ the same conditions (2.10). Hence it is sufficient to prove the lemma locally. The general case follows by a partition of unity. Assume that the boundary $S$ in the coordinates $\{y\}$ is given by the equation

$$
y_{3}=\varphi\left(y_{1}, y_{2}\right) \equiv \varphi\left(y^{\prime}\right), \quad \varphi(0)=0
$$

where $\varphi \in H^{5 / 2}$. Let $\bar{\tau}_{1}=\left(-n_{3}, 0, n_{1}\right) /\left(n_{1}^{2}+n_{3}^{2}\right)^{1 / 2}, \bar{\tau}_{2}=\left(0,-n_{3}, n_{2}\right) /\left(n_{2}^{2}+n_{3}^{2}\right)^{1 / 2}$ in (2.10), where $\bar{n}=\left(n_{1}, n_{2}, n_{3}\right)$ and $|\bar{n}|=1$. Then by $(2.10)$ the vector $w$ defined in the 
domain $\left\{y: y_{3}<\varphi\left(y^{\prime}\right)\right\}$ satisfies

$$
\begin{aligned}
& w_{1}=0, \quad w_{2}=0, \quad w_{3}=a / n_{3}, \quad \frac{\partial w_{3}}{\partial y_{3}}=0 \quad \text { on } S, \\
& \frac{\partial w_{1}}{\partial y_{3}}=\frac{b_{1}\left(n_{2}^{2}-n_{3}^{2}\right)\left(n_{1}^{2}+n_{3}^{2}\right)^{1 / 2}-b_{2} n_{1} n_{2}\left(n_{2}^{2}+n_{3}^{2}\right)^{1 / 2}}{n_{3}^{2}\left(n_{3}^{2}-n_{1}^{2}-n_{2}^{2}\right)}-\frac{\partial}{\partial y_{1}}\left(\frac{a}{n_{3}}\right) \text { on } S, \\
& \frac{\partial w_{2}}{\partial y_{3}}=\frac{b_{2}\left(n_{1}^{2}-n_{3}^{2}\right)\left(n_{2}^{2}+n_{3}^{2}\right)^{1 / 2}-b_{1} n_{1} n_{2}\left(n_{1}^{2}+n_{2}^{2}\right)^{1 / 2}}{n_{3}^{2}\left(n_{3}^{2}-n_{1}^{2}-n_{2}^{2}\right)}-\frac{\partial}{\partial y_{2}}\left(\frac{a}{n_{3}}\right) \text { on } S .
\end{aligned}
$$

Since $\bar{n}=\left(-\frac{\partial \varphi}{\partial y_{1}},-\frac{\partial \varphi}{\partial y_{2}}, 1\right) /\left(1+\left|\nabla^{\prime} \varphi\right|^{2}\right)^{1 / 2}$, the expressions in (2.12) are defined for a sufficiently small neighbourhood of the point $y^{\prime}=0$. Now the existence of $w$ satisfying (2.12) and the inequality $\|w\|_{3} \leqq c\left(\|a\|_{5 / 2, S}+\|h\|_{3 / 2, S}\right)$ $\leqq c\left(\|a\|_{5 / 2, S}+\|b\|_{3 / 2, S}\right)$ follows from the results of [25] (see Chap. 7) and from embedding theorems.

Let $B_{R}=\{x:|x|<R\}$ be a ball in $\mathbb{R}^{3}$. Consider the following problem:

$$
\begin{gathered}
\zeta+\operatorname{div}(u \zeta)=G \quad \text { in } B_{R}, \\
\left.\zeta\right|_{\partial B_{R}}=0, \quad \partial \zeta /\left.\partial n\right|_{\partial B_{R}}=0 .
\end{gathered}
$$

Lemma 2.5. Let $G \in H_{0}^{2}\left(B_{R}\right), u \in H^{3}\left(B_{R}\right),\left.u \cdot \bar{n}\right|_{\partial B_{R}}=0$ and $\|u\|_{3, B_{R}}<\gamma$, where $\gamma$ is a sufficiently small constant. Then problem (2.13) has a unique solution $\zeta \in H_{0}^{2}\left(B_{R}\right)$ such that

$$
\|\zeta\|_{2} \leqq c\|G\|_{2} .
$$

Proof. Let $\zeta_{\varepsilon} \in H^{4}\left(B_{R}\right) \cap H_{0}^{2}\left(B_{R}\right)$ be a solution of

$$
\begin{gathered}
\varepsilon \Delta^{2} \zeta_{\varepsilon}+\zeta_{\varepsilon}+\operatorname{div}\left(u \zeta_{\varepsilon}\right)=G \quad \text { in } B_{R} \\
\left.\zeta_{\varepsilon}\right|_{\partial B_{R}}=0,\left.\quad\left(\partial \zeta_{\varepsilon} / \partial n\right)\right|_{\partial B_{R}}=0
\end{gathered}
$$

where $\varepsilon$ is a positive constant. Multiplying both sides of $(2.15)_{1}$ by $\zeta_{\varepsilon}$, intergrating over $B_{R}$ and using $\|u\|_{3}<\gamma$ with $\gamma$ small enough, one easily gets

$$
\varepsilon\left\|\Delta \zeta_{\varepsilon}\right\|_{0}^{2}+\left\|\zeta_{\varepsilon}\right\|_{0}^{2} \leqq c\|G\|_{0}^{2}
$$

Analogously, multiplying (2.15) by $\Delta^{2} \zeta_{\varepsilon}$ gives

$$
\varepsilon \int_{B_{R}}\left|\Delta^{2} \zeta_{\varepsilon}\right|^{2} d x+\int_{B_{R}}\left|\Delta \zeta_{\varepsilon}\right|^{2} d x \leqq\|G\|_{2}\left\|\Delta \zeta_{\varepsilon}\right\|_{0}+\left|\int_{B_{R}} \operatorname{div}\left(u \zeta_{\varepsilon}\right) \Delta \zeta_{\varepsilon}^{2} d x\right| .
$$

Consider the last integral on the right-hand side of (2.17):

$$
J=\int_{B_{R}} \operatorname{div}\left(u \zeta_{\varepsilon}\right) \Delta^{2} \zeta_{\varepsilon} d x=\int_{B_{R}} \Delta\left(\operatorname{div} u \zeta_{\varepsilon}+u \nabla \zeta_{\varepsilon}\right) \Delta \zeta_{\varepsilon} d x \equiv J_{1}+J_{2} .
$$

Embedding theorems give

$$
\begin{gathered}
\left|J_{1}\right| \leqq c\|u\|_{3}\left(\left|\zeta_{\varepsilon}\right|_{\infty}+\left|\nabla \zeta_{\varepsilon}\right|_{4}+\left\|\Delta \zeta_{\varepsilon}\right\|_{0}\right)\left\|\Delta \zeta_{\varepsilon}\right\|_{0}, \\
\left|J_{2}\right| \leqq c\|u\|_{3}\left(\left|\nabla \zeta_{\varepsilon}\right|_{4}+\left\|\nabla \nabla \zeta_{\varepsilon}\right\|_{0}+\left\|\Delta \zeta_{\varepsilon}\right\|_{0}\right)\left\|\Delta \zeta_{\varepsilon}\right\|_{0} .
\end{gathered}
$$


Moreover, by interpolation inequalities,

$$
\left|\nabla \zeta_{\varepsilon}\right|_{p} \leqq c \delta\left\|\Delta \zeta_{\varepsilon}\right\|_{0}+c(\delta)\left\|\zeta_{\varepsilon}\right\|_{0}, \quad \delta \in(0,1), \quad p=4, \infty .
$$

Hence, for small $\gamma,(2.16)-(2.19)$ imply

$$
\varepsilon\left\|\Delta^{2} \zeta_{\varepsilon}\right\|_{0}^{2}+\left\|\Delta \zeta_{\varepsilon}\right\|_{0}^{2}+\left\|\zeta_{\varepsilon}\right\|_{0}^{2} \leqq c\|G\|_{2}^{2} .
$$

The last estimate yields the existence of a subsequence $\zeta_{\varepsilon}$ such that $\varepsilon\left\|\Delta^{2} \zeta_{\varepsilon}\right\|_{0} \rightarrow 0$, $\zeta_{\varepsilon} \rightarrow \zeta$ weakly in $H_{0}^{2}\left(B_{R}\right)$ and strongly in $H^{1}\left(B_{R}\right)$ as $\varepsilon \rightarrow 0$. Passing to the limit in (2.15) shows that $\zeta$ is a solution of (2.13). Estimate (2.14) follows from (2.20).

Consider now the problem

$$
\lambda+\operatorname{div}(v \lambda)=g
$$

in a bounded domain $\Omega$ with $S \in C^{2}$. Assume that

$$
v \in H^{3}(\Omega),\left.v \cdot \bar{n}\right|_{S}=0, \quad g \in H^{2}(\Omega) .
$$

Lemma 2.6. Let conditions (2.22) be satisfied and let $\|v\|_{3}<\gamma$, where $\gamma$ is sufficiently small. Then Eq. (2.21) has a unique solution $\lambda \in H^{2}(\Omega)$ satisfying

$$
\|\lambda\|_{2} \leqq c\|g\|_{2} \text {. }
$$

Proof. Let $R$ be large enough to satisfy $\bar{\Omega} \subset B_{R}$. We can find extensions $u$ and $G$ of $v$ and $g$ such that

$$
\begin{gathered}
G \in H_{0}^{2}\left(B_{R}\right), \quad u \in H^{3}\left(B_{R}\right),\left.\quad u \cdot \bar{n}\right|_{\partial B_{R}}=0 \\
\|G\|_{2, B_{R}} \leqq c\|g\|_{2, \Omega}, \quad\|u\|_{3, B_{R}} \leqq c\|v\|_{3, \Omega} .
\end{gathered}
$$

Let $\zeta$ be the solution of (2.13) with these $u$ and $G$. By (2.14) and (2.24), $\zeta \in H_{0}^{2}\left(B_{R}\right)$ and

$$
\|\zeta\|_{2, B_{R}} \leqq c\|g\|_{2, \Omega} .
$$

Using the trace operator to Eq. $(2.13)_{1}$ one can see that $\lambda=\left.\zeta\right|_{\Omega}$ satisfies (2.21). Moreover,

$$
\|\lambda\|_{2, \Omega} \leqq c\|\zeta\|_{2, B_{R}} \leqq c\|g\|_{2, \Omega} .
$$

It was proved in [7] that problem (2.21), (2.22) has a unique solution $\lambda \in L_{2}(\Omega)$ for every $g \in L_{2}(\Omega)$. Hence the lemma is proved.

Remark 2.2. The condition $\int_{\Omega} g d x=0$ implies that $\int_{\Omega} \lambda d x=0$.

\section{On the Stokes Problem in a Prescribed Domain}

Let $\Omega \subset \mathbb{R}^{3}$ be a bounded domain with boundary $S$. Consider the following boundary value problem for the Stokes equations:

$$
\begin{gathered}
-\mu \Delta v-v \nabla \operatorname{div} v+\nabla p=f, \quad \operatorname{div} v=g \quad \text { in } \Omega, \\
\left.v \cdot \bar{n}\right|_{S}=0,\left.\quad\left(T^{\prime}(v) \bar{n}-\left(\bar{n} \cdot T^{\prime}(v) \cdot \bar{n}\right) \bar{n}\right)\right|_{S}=0 \quad \text { on } S,
\end{gathered}
$$


where $g$ satisfies the condition

$$
\int_{\Omega} g d x=0 .
$$

For a bounded domain $\Omega$ obtained by rotation about vector $B$, the function $f$ must satisfy the condition

$$
\int_{\Omega} f u_{0} d x=0
$$

where $u_{0}=B \times x$. Note that (3.3), (3.4) are necessary conditions for the existence of a classical solution to (3.1), (3.2) (see [47]).

By a generalized solution to problem (3.1), (3.2) we mean a vector $v \in H(\Omega)$ and a function $p \in \hat{L}_{2}(\Omega)=\left\{p \in L_{2}(\Omega):(p, 1)_{\Omega}=0\right\}$ which satisfy the integral identity

$$
\frac{\mu}{2} E(v, \eta)-(p, \operatorname{div} \eta)_{\Omega}=(f, \eta)_{\Omega}-(v-\mu)(g, \operatorname{div} \eta)_{\Omega}
$$

for all $\eta \in H(\Omega)$, and such that $\operatorname{div} v=g$. Here $E(v, \eta)$ is defined by (2.1). The problem

$$
\operatorname{div} \tilde{v}=g,\left.\quad \tilde{v} \cdot \bar{n}\right|_{S}=0
$$

has a solution such that $\tilde{v} \in H^{1}(\Omega)$ and

$$
\|\tilde{v}\|_{1, \Omega} \leqq c\|g\|_{0, \Omega} \text {. }
$$

If $\eta$ in (3.5) is divergence-free, then $u=v-\tilde{v}$ satisfies

$$
\frac{\mu}{2} E(u, \eta)=(f, \eta)_{\Omega}-\frac{\mu}{2} E\left(\tilde{v}^{\prime}, \eta\right) .
$$

By the Riesz theorem, for $f, g \in L_{2}(\Omega)$ satisfying (3.3), (3.4) there exists a solution to (3.8) such that $u \in\{v \in H(\Omega): \operatorname{div} v=0\}$ and

$$
\|u\|_{1, \Omega} \leqq \frac{c}{\mu}\|f\|_{0, \Omega}+c\|g\|_{0, \Omega} .
$$

Having found $v=u+\tilde{v}$, identity (3.5) implies that there exists $p \in \hat{L}_{2}(\Omega)$ (see $[21,47])$. Let $\eta$ be a solution to (3.6) for $g=p$. Then from (3.5) we get the estimate

$$
\|p\|_{0, \Omega} \leqq c\|f\|_{0, \Omega}+c(\xi+\mu)\|g\|_{0, \Omega} .
$$

Therefore we have shown (see $[21,47]$, in reality we repeat the considerations from [47]).

Theorem 3.1. Let $S \in$ Lip. Then for any $g, f \in L_{2}(\Omega)$ satisfying (3.3), (3.4), problem (3.1), (3.2) has a unique generalized solution $(v, p) \in H(\Omega) \times \hat{L}_{2}(\Omega)$ such that

$$
\|v\|_{1, \Omega}+\|p\|_{0, \Omega} \leqq c\left(\|f\|_{0, \Omega}+\|g\|_{0, \Omega}\right) .
$$

Let us now establish the differential properties of the generalized solution.

Theorem 3.2. Let $f \in H^{s}(\Omega), g \in H^{1+s}(\Omega), S \in H^{5 / 2+s}$ and let (3.3), (3.4) be satisfied. Then the generalized solution $(v, p)$ of problem (3.1), (3.2) belongs to $H^{2+s}(\Omega)$ $\times H^{1+s}(\Omega)$ and

$$
\|v\|_{2+s, \Omega}+\|p\|_{1+s, \Omega} \leqq c\left(\|f\|_{s, \Omega}+\|g\|_{1+s, \Omega}\right), \quad s=0,1 .
$$


Remark 3.1. In the case $S \in C^{3}$ the same result was proved in [47]. Theorem 3.2 was shown by Solonnikov (private communication). For the reader's convenience we present the proof in the Appendix.

Let us now consider the nonhomogeneous Stokes problem corresponding to (3.1), (3.2). Hence instead of (3.2) we have

$$
\left.v \cdot \bar{n}\right|_{S}=a,\left.\quad\left(T^{\prime}(v) \bar{n}-\left(\bar{n} T^{\prime}(v) \bar{n}\right) \bar{n}\right)\right|_{S}=b .
$$

Using Lemma 2.4 and Theorem 3.2 one has

Theorem 3.3. Let $f \in H^{1}(\Omega), g \in H^{2}(\Omega), S \in H^{7 / 2}, a \in H^{5 / 2}, b \in H^{3 / 2}$, let (3.4) be statisfied, and let

$$
\int_{S} a d \sigma=\int_{\Omega} g d x
$$

Then there exists a solution (v,p) of problem (3.1), (3.14) such that $v \in H^{3}(\Omega)$, $p \in H^{2}(\Omega)$ and

$$
\|v\|_{3}+\|p\|_{2} \leqq c\left(\|f\|_{1}+\|g\|_{2}+\|a\|_{5 / 2, S}+\|b\|_{3 / 2, S}\right) .
$$

Remark 3.2. Suppose the right-hand side functions of problem (3.1), (3.13) do not depend on $\varphi$ (in the cylindrical coordinates), symmetry conditions (5.4), (3.14), (3.4) are satisfied and $\Omega$ is cylindrically symmetric which satisfies symmetry condition (5.1). Then there exists a cylindrically symmetric solution to the problem (3.1), (3.13), which satisfies symmetry conditions (5.6).

\section{Existence and Uniqueness for Compressible Navier-Stokes Equations}

In this section we prove the existence and uniqueness of solutions to the compressible Navier-Stokes system in a bounded domain with prescribed boundary. First we consider the following linear problem which follows from problem (1.1):

$$
\begin{gathered}
\bar{A} v+p_{1} \nabla \eta=K \quad \text { in } \Omega \\
v \cdot \bar{n}=0 \quad \text { on } S, \\
\bar{\tau}_{k} T^{\prime}(v) \bar{n}=0 \quad \text { on } S, k=1,2,
\end{gathered}
$$

where $\bar{A}=-\frac{\mu}{\bar{\varrho}} \Delta-\frac{v}{\bar{\varrho}} \nabla \operatorname{div}, \eta=\varrho-\bar{\varrho}, p_{1}=\frac{p_{\varrho}(\bar{\varrho})}{\varrho}>0, p_{\varrho}=\frac{\partial p}{\partial \varrho}, \bar{\varrho}$ is defined in Sect. 1 and

$$
\int_{\Omega} \eta d x=0
$$

We also consider the following equation

where

$$
\operatorname{div}(w \eta)+\bar{\varrho} \operatorname{div} v=G,
$$

$$
w \cdot \bar{n}=0 \quad \text { on } S .
$$

First we obtain an a priori estimate for solutions of the above problem. To do this we follow the methods of [62] and [63] restricted to the stationary case. 
To obtain the a priori estimate we assume

$$
\xi \geqq \frac{2}{3} \mu \text {. }
$$

Lemma 4.1. Let $(v, \eta) \in H^{3}(\Omega) \times H^{2}(\Omega)$ be a solution of $(4.1) \div(4.5), w \in H^{3}(\Omega)$ and let (4.6) be satisfied. Then

where

$$
\|v\|_{2}^{2}+\|\eta\|_{1}^{2} \leqq \varepsilon_{1}\|v\|_{3}^{2}+c\|w\|_{3}^{2}\|\eta\|_{0}^{2}+Y_{1}
$$

$$
Y_{1}=c\left(\|K\|_{0}^{2}+\|G\|_{0}^{2}\right)
$$

and $\varepsilon_{1}$ can be made arbitrarily small.

Proof. Multiplying (4.1) by $v$, (4.4) by $\frac{p_{1}}{\bar{\varrho}} \eta$, integrating over $\Omega$ and adding these expressions one gets

$$
\int_{\Omega}\left(\bar{A} v \cdot v+p_{1} \operatorname{div}(v \eta)+\frac{p_{1}}{\bar{\varrho}} \operatorname{div}(w \eta) \eta\right) d x=\int_{\Omega}\left(K v+\frac{p_{1}}{\bar{\varrho}} G \eta\right) d x .
$$

Using (4.2) the first term on the left-hand side is

$$
\begin{aligned}
\int_{\Omega} \bar{A} v \cdot v d x= & \frac{1}{\varrho} \int_{\Omega}-\left[\mu\left(\frac{\partial v_{i}}{\partial x_{j}}+\frac{\partial v_{j}}{\partial x_{i}}\right)+\left(\xi-\frac{2}{3} \mu\right) \delta_{i j} \operatorname{div} v\right]_{x_{j}} v_{i} d x \\
= & \frac{1}{\bar{\varrho}} \int_{S}-\left[\mu\left(\frac{\partial v_{i}}{\partial x_{j}}+\frac{\partial v_{j}}{\partial x_{i}}\right)+\left(\xi-\frac{2}{3} \mu\right) \delta_{i j} \operatorname{div} v\right] n_{j} v_{i} d s \\
& +\frac{\mu}{\bar{\varrho}} \int_{\Omega}\left(\frac{\partial v_{i}}{\partial x_{j}} \frac{\partial v_{i}}{\partial x_{j}}+\frac{\partial v_{i}}{\partial x_{j}} \frac{\partial v_{j}}{\partial x_{i}}\right) d x \\
& +\frac{1}{\bar{\varrho}}\left(\xi-\frac{2}{3} \mu\right) \int_{\Omega}(\operatorname{div} v)^{2} d x=\frac{\mu}{2 \bar{\varrho}} E(v, v) \\
& +\frac{1}{\varrho}\left(\xi-\frac{2}{3} \mu\right) \int_{\Omega}(\operatorname{div} v)^{2} d x .
\end{aligned}
$$

The second term on the left-hand side of (4.9) vanishes because of the Stokes formula and (4.2) $)_{1}$. Finally the third term on the left-hand side of (4.9) is estimated by

$$
\int_{\Omega} \operatorname{div}(w \eta) \eta d x=\int_{\Omega} \frac{\eta^{2}}{2} \operatorname{div} w d x \leqq \varepsilon_{2}\|\eta\|_{0}^{2}+\frac{c}{\varepsilon_{2}}\|w\|_{3}^{2}\|\eta\|_{0}^{2},
$$

where the embedding $H^{3}(\Omega) \subset W_{\infty}^{1}(\Omega), \Omega \subset \mathbb{R}^{3}$, has been used.

Summarizing

where

$$
E(v, v) \leqq c X_{1}+\left(\varepsilon_{2}+\varepsilon_{3}\right)\|\eta\|_{0}^{2}+\frac{c}{\varepsilon_{2}}\|w\|_{3}^{2}\|\eta\|_{0}^{2}
$$

$$
X_{1}=\|K\|_{-1}^{2}+\frac{1}{\varepsilon_{3}}\|G\|_{0}^{2} .
$$


Using Theorem 3.2 to the Stokes problem

$$
\begin{gathered}
\bar{A} v+p_{1} \nabla \eta=K \quad \text { in } \Omega, \\
\operatorname{div} v=\operatorname{div} v \quad \text { in } \Omega, \\
v \cdot \bar{n}=0 \quad \text { on } S, \\
\bar{\tau} \cdot T^{\prime}(v) \bar{n}=0 \quad \text { on } S,
\end{gathered}
$$

and (4.3) gives

$$
\|v\|_{2}^{2}+\|\eta\|_{1}^{2} \leqq c\left(\|\operatorname{div} v\|_{1}^{2}+\|K\|_{0}^{2}\right) .
$$

By the interpolation inequality

$$
\|\operatorname{div} v\|_{1}^{2} \leqq \varepsilon_{4}\|v\|_{3}^{2}+\frac{c}{\varepsilon_{4}}\|v\|_{1}^{2}
$$

estimates (4.10), (4.13) and the Korn inequality one obtains (4.7) for sufficiently small $\varepsilon_{2}, \varepsilon_{3}$ and $\varepsilon_{1}=\varepsilon_{4}$.

Now we prove the main estimate for problem (4.1), (4.2), (4.4).

Lemma 4.2. A solution $(v, \eta) \in H^{3}(\Omega) \times H^{2}(\Omega)$ to problem (4.1), (4.2), (4.4) with conditions (4.3) and (4.6) satisfies the estimate

where

$$
\|v\|_{3}^{2}+\|\eta\|_{2}^{2} \leqq c Y_{2}
$$

$$
Y_{2} \equiv\|K\|_{1}^{2}+\|G\|_{2}^{2}+C(S)\|w\|_{3}^{2}\|\eta\|_{2}^{2},
$$

$w \in H^{3}(\Omega)$ and satisfies (4.5).

Proof. We consider problem $(4.1) \div(4.4)$ locally. We restrict our attention to a neighbourhood of the boundary, because the considerations in interior subdomains are simpler.

We need some notation. Let us introduce a partition of unity $\left(\left\{\widetilde{\Omega}_{i}\right\},\left\{\zeta_{i}\right\}\right)$. Let $\widetilde{\Omega}$ be one of the $\widetilde{\Omega}_{i}$ 's and $\zeta(x)=\zeta_{i}(x)$ the corresponding function. Let $\tilde{\omega}$ be such that $\overline{\tilde{\omega}} \subset \tilde{\Omega}$ and $\zeta(x)=1$ for $x \in \tilde{\omega}$. Considering a neighbourhood of a boundary we assume that $\widetilde{\widetilde{\Omega}} \cap S \neq \emptyset, \overline{\tilde{\omega}} \cap S \neq \emptyset$. Let $\beta \in \overline{\tilde{\omega}} \cap S=\widetilde{S} \equiv \widetilde{\Omega} \cap S$. Introduce local coordinates $\{y\}$ connected with $\{x\}$ by the relation

$$
y_{k}=\sum_{l=1}^{3} \alpha_{k l}\left(x_{l}-\beta_{l}\right), \quad \alpha_{3 k}=n_{k}(\beta), \quad k=1,2,3,
$$

where $\alpha_{k l}$ is a constant orthogonal matrix such that $\tilde{S}$ is determined by the equation $y_{3}=F\left(y_{1}, y_{2}\right), F \in H^{7 / 2}$, and $\widetilde{\Omega}=\left\{y:\left|y_{i}\right|<d, i=1,2, F\left(y^{\prime}\right)<y_{3}<F\left(y^{\prime}\right)+d\right.$, $\left.y^{\prime}=\left(y_{2}, y_{2}\right)\right\}$. Simultaneously we introduce a new vector $u^{\prime}$ (where $u$ replaces $v, \eta$ and so on) by the formula

$$
u_{i}^{\prime}(y)=\left.\sum_{j=1}^{3} \alpha_{i j} u_{j}(x)\right|_{x=x(y)}
$$

and scalar $p^{\prime}$ defined as $p^{\prime}(y)=\left.p(x)\right|_{x=x(y)}$, where $x=x(y)$ is the inverse transformation to (4.17). Further, we introduce new variables by

$$
z_{i}=y_{i}, \quad i=1,2, \quad z_{3}=y_{3}-\tilde{F}\left(y_{1}, y_{2}, y_{3}\right), \quad y \in \tilde{\Omega},
$$


which will be denoted by $z=\Phi(y)$, where $\widetilde{F}$ is an extension of $F$, so $\widetilde{F} \in H^{4}(\Omega)$. Let $Q=\Phi(\widetilde{\Omega})=\left\{z:\left|z_{i}\right|<d, \quad i=1,2,0<z_{3}<d\right\}$ and $\hat{u}(z)=\left.u^{\prime}(y)\right|_{y=\Phi^{-1}(z)}$. Finally, set $\hat{S}=\Phi(\widetilde{S})$.

We also asume that $\hat{\nabla}_{k}=\left.\frac{\partial z_{i}}{\partial y_{k}} \nabla_{z_{i}}\right|_{y=\Phi-1(z)}$.

Moreover, we introduce the operator $\delta_{h}^{l} a(z)=h^{-1}\left[a\left(z_{1}, \ldots, z_{l}+h\right.\right.$, $\left.\left.z_{l+1}, \ldots, z_{n}\right)-a(z)\right], l \leqq 2, n=3$, which satisfies the formulas $\delta_{n}^{l}(a \cdot b)=a_{h}^{l} \delta_{h}^{l} b+\delta_{h}^{l} a b$, $\left(\delta_{h}^{l} a, b\right)_{Q}=-\left(a, \delta_{-h}^{l} b\right)_{Q}$, where $a_{h}^{l}(z)=a\left(z_{1}, \ldots, z_{l-1}, z_{l}+h, z_{l+1}, \ldots, z_{n}\right)$.

Using this notation we write Eqs. (4.1), (4.4) in the form

$$
\begin{gathered}
\hat{\bar{A}} \tilde{v}+p_{1} \hat{\nabla} \tilde{\eta}=\tilde{K}+k_{1} \quad \text { in } Q, \\
\hat{\nabla} \cdot(\hat{w} \tilde{\eta})+\bar{\varrho} \widehat{\operatorname{div}} \tilde{v}=\tilde{G}+k_{2} \quad \text { in } Q,
\end{gathered}
$$

where $\tilde{v}=\hat{v} \widehat{\zeta}, \tilde{\eta}=\hat{\eta} \widehat{\zeta}, \tilde{K}=\hat{K} \hat{\zeta}, \tilde{G}=\hat{G} \widehat{\zeta}, \widehat{\bar{A}}=-\frac{\mu}{\bar{\varrho}} \hat{\nabla}^{2}-\frac{v}{\bar{Q}} \hat{\nabla} \widehat{\operatorname{div}}, \widehat{\operatorname{div}}=\hat{\nabla} \cdot$,

$$
\begin{aligned}
& k_{1}=p_{1} \hat{\eta} \hat{\nabla} \hat{\zeta}-\bar{\mu}\left(2 \hat{\nabla_{k}} \hat{v} \hat{V}_{k} \hat{\zeta}+\hat{v} \hat{\Delta} \hat{\zeta}\right)-\bar{v}\left(\widehat{\operatorname{div}} \hat{v} \hat{\nabla} \hat{\zeta}+\hat{\nabla} \hat{v}_{k} \hat{\nabla_{k}} \hat{\zeta}+\hat{v}_{k} \hat{\nabla} \hat{\nabla_{k}} \hat{\zeta}\right), \\
& k_{2}=\hat{\eta} \hat{w} \hat{\nabla} \hat{\zeta}+\bar{\varrho} \hat{v} \hat{\nabla} \hat{\zeta} .
\end{aligned}
$$

Moreover, we set $z_{\alpha}=\tau_{\alpha}, \alpha=1,2, z_{3}=n$.

By taking the operator $\hat{\nabla}$ of (4.20) multipled by $\frac{\bar{\mu}+\bar{v}}{\bar{\varrho}}$ and adding the result to (4.19) one has

$$
p_{1} \hat{\nabla} \tilde{\eta}=\bar{\mu}(\hat{\Delta} \tilde{v}-\hat{\nabla} \widehat{\operatorname{div}} \tilde{v})-\frac{\bar{\mu}+\bar{v}}{\bar{\varrho}} \hat{\nabla} \hat{\nabla} \cdot(\hat{w} \tilde{\eta})+\widetilde{K}+\frac{\bar{\mu}+\bar{v}}{\bar{\varrho}} \hat{\nabla} \tilde{G}+k_{3},
$$

where $k_{3} \equiv \frac{\bar{\mu}+\bar{v}}{\bar{\varrho}} \hat{\nabla} k_{2}+k_{1}$. Differentiating (4.19), (4.20) once with respect to $\tau$, applying the operator $\delta_{n}^{l}, l<3$, multiplying by $\delta_{h}^{l} \tilde{v}_{\tau}, \frac{p_{1}}{\bar{\varrho}} \delta_{h}^{l} \tilde{\eta}_{\tau}$, respectively, integrating over $Q$ and summing one gets

$$
\begin{aligned}
\int_{\Omega}\left[\delta_{h}^{l}(\hat{\bar{A}} \tilde{v})_{\tau} \delta_{h}^{l} \tilde{v}_{\tau}\right. & \left.+\frac{p_{1}}{\bar{\varrho}} \delta_{h}^{l}(\hat{\nabla} \cdot(\hat{w} \tilde{\eta})){ }_{\tau} \delta_{h}^{l} \tilde{\eta}_{\tau}+p_{1} \delta_{h}^{l}(\hat{\nabla} \tilde{\eta})_{\tau} \delta_{h}^{l} \tilde{v}_{\tau}+p_{1} \delta_{h}^{l}(\widehat{\operatorname{div}} \tilde{v})_{\tau} \delta_{h}^{l} \tilde{\eta}_{\tau}\right] d z \\
= & \int_{\Omega}\left[\left(\delta_{h}^{l} \tilde{K}_{\tau}+\delta_{h}^{l} k_{1},{ }_{\tau}\right) \delta_{h}^{l} \tilde{v}_{\tau}+\frac{p_{1}}{\bar{\varrho}}\left(\delta_{h}^{l} \tilde{G}_{\tau}+\delta_{h}^{l} k_{2},{ }_{\tau}\right) \delta_{h}^{l} \tilde{\eta}_{\tau}\right] d z
\end{aligned}
$$

Now we consider each term on the left-hand side of (4.23) separately. For the second term we have

$$
\begin{aligned}
\int_{\Omega} \delta_{h}^{l}(\hat{\nabla} \cdot(\hat{w} \tilde{\eta})),{ }_{\tau} \delta_{h}^{l} \tilde{\eta}_{\tau} d z= & \int_{\Omega}\left(\hat{w} \hat{z}_{y}\right)_{h}^{l} \cdot \nabla_{z} \delta_{h}^{l} \tilde{\eta}_{\tau} \delta_{h}^{l} \tilde{\eta}_{\tau} d z \\
& +\int_{\Omega}\left\{\left[\delta_{h}^{l}\left(\hat{w} \hat{z}_{y}\right) \nabla_{z} \tilde{\eta}_{\tau}+\delta_{h}^{l}\left(\hat{z}_{y} \nabla_{z} \cdot \hat{w} \tilde{\eta}\right),_{\tau}\right] \delta_{h}^{l} \tilde{\eta}_{\tau}\right\} d z
\end{aligned}
$$

The first term on the right-hand side of (4.24) gives

$$
\begin{gathered}
\frac{1}{2} \int_{\partial Q}\left(\hat{w}_{i} \hat{z}_{k y_{i}}\right)_{h}^{l} \hat{n}_{k}\left(\delta_{h}^{l} \tilde{\eta}_{\tau}\right)^{2}-\frac{1}{2} \int_{Q}\left(\delta_{h}^{l} \tilde{\eta}_{\tau}\right)^{2}\left(\hat{w}_{i} \hat{z}_{k y_{i}}\right)_{h, z_{k}}^{l} d z \leqq \varepsilon\|\tilde{\eta}\|_{2, Q}^{2} \\
+\frac{c}{\varepsilon} P\left(\left\|\hat{z}_{y}\right\|_{3, Q}\right)\|\hat{w}\|_{3, Q}^{2}\|\hat{\eta}\|_{2, Q^{\prime}}^{2}
\end{gathered}
$$


where $P$ is a polynomial and the boundary term vanishes because

$$
\Phi^{-1}\left(w_{i} z_{k y_{\imath}}\right)_{h}^{l} n_{k}|\hat{s}=w \cdot \bar{n}|_{S}=0 \text {. }
$$

In obtaining estimate (4.25) we have used the facts that

$$
\left\|\delta_{h}^{l} u\right\|_{0, Q} \leqq c\left\|u_{\tau}\right\|_{0, Q} \text { for } u \in H^{1}(Q), l<3, h \text {-arbitrary }
$$

and $z=z(y) \in H^{4}(\Omega)$, so

$$
\left|\left(\hat{w}_{i} \hat{z}_{k y_{i}}\right)_{h z_{k}}^{l}\right|_{\infty, Q} \leqq c P\left(\left\|\hat{z}_{y}\right\|_{3, Q}\right)\|\hat{w}\|_{3, Q},
$$

where theorems of embedding were used. Below using theorems of embedding similar considerations will be done also however this will not be mentioned explicitly.

The other terms in (4.24) are estimated by

$$
P\left(\left\|\hat{z}_{y}\right\|_{3, Q}\right)\left\|\hat{z}_{y}\right\|_{3, Q}\|\hat{w}\|_{3, Q}\|\tilde{\eta}\|_{2, Q}\left\|\tilde{\eta}_{\tau \tau}\right\|_{0, Q} \leqq \varepsilon\|\hat{\eta}\|_{2, Q}^{2}+\frac{c}{\varepsilon} P\left(\left\|\hat{z}_{y}\right\|_{3, Q}\right)\|\hat{w}\|_{3, Q}^{2}\|\hat{\eta}\|_{2, Q}^{2}
$$

By (4.26) the third and fourth terms on the left of (4.23) are estimated by

$$
P\left(\left\|\hat{z}_{y}\right\|_{3, Q}\right)\left\|\hat{z}_{y}\right\|_{3, Q}\left(\|\tilde{\eta}\|_{1, Q}+\left\|\tilde{\eta}_{\tau}\right\|_{1, Q}\right)\|\tilde{v}\|_{2, Q}
$$

if we integrate by parts and use the fact that the boundary term $\int_{\partial Q}\left(\hat{n}_{k}\left(\hat{z}_{k y_{i}}\right)_{h}^{l} \delta_{h}^{l} \tilde{v}_{i}\right)_{\tau} \delta_{h}^{l} \tilde{\eta}_{\tau}$
vanishes.

The first term on the left-hand side of (4.23) is

$$
\frac{\mu}{2 \bar{\varrho}} \hat{E}\left(\delta_{h}^{l} \tilde{v}_{\tau}, \delta_{h}^{l} \tilde{v}_{\tau}\right)+\frac{1}{\bar{\varrho}}\left(\xi-\frac{2}{3} \mu\right) \int_{Q}\left(\widehat{\operatorname{div}} \delta_{h}^{l} \tilde{v}_{\tau}\right)^{2} d z+\{\},
$$

where $\hat{E}(u, v)=\int_{Q}\left(\hat{\nabla}_{i} u_{j}+\hat{\nabla}_{j} u_{i}\right)\left(\hat{\nabla}_{i} v_{j}+\hat{\nabla}_{j} v_{i}\right)$ and \{\} can be estimated by

$$
\begin{gathered}
P\left(\left\|\hat{z}_{y}\right\|_{3, Q}\right)\left\|\hat{z}_{y}\right\|_{2, Q}\|\hat{v}\|_{3, Q}\|\hat{v}\|_{2, Q} \\
\text { Summarizing, } \\
\frac{\mu}{2} \hat{E}\left(\delta_{h}^{l} \tilde{v}_{\tau}, \delta_{h}^{l} \tilde{v}_{\tau}\right)+\left(\xi-\frac{2}{3} \mu\right) \int_{Q}\left(\widehat{\operatorname{div}} \delta_{h}^{l} \tilde{v}_{\tau}\right)^{2} d z \leqq c\left(X_{2}+X_{3}\right)+\varepsilon\left(\|\hat{\eta}\|_{2, Q}^{2}+\|\hat{v}\|_{3, Q}^{2}\right)
\end{gathered}
$$

where

$$
\begin{aligned}
& X_{2} \equiv P\left(\left\|\hat{z}_{y}\right\|_{3, Q}\right)\left(\|\hat{v}\|_{2, Q}^{2}+\|\hat{\eta}\|_{1, Q}^{2}\right), \\
& X_{3} \equiv\|\tilde{K}\|_{1, Q}^{2}+\|\tilde{G}\|_{2, Q}^{2}+P\left(\left\|\hat{z}_{y}\right\|_{3, Q}\right)\|\hat{\eta}\|_{2, Q}^{2}\|\hat{w}\|_{3, Q}^{2},
\end{aligned}
$$

and the following inequalities have been used:

$$
\begin{aligned}
& \int_{Q} \delta_{h}^{l} k_{1, \tau} \delta_{h}^{l} \tilde{v}_{\tau} d z \leqq \varepsilon\|\hat{v}\|_{3, Q}^{2}+c\left(\|\hat{v}\|_{2, Q}^{2}+\|\hat{\eta}\|_{1, Q}^{2}\right), \\
& \int_{Q} \delta_{h}^{l} k_{2, \tau} \delta_{h}^{l} \tilde{\eta}_{\tau} d z \leqq \varepsilon\left\|\tilde{\eta}_{\tau \tau}\right\|_{0, Q}^{2}+c P\left(\left\|\hat{z}_{y}\right\|_{3, Q}\right)\left(\|\hat{\eta}\|_{3, Q}^{2}\|\hat{w}\|_{3, Q}^{2}+\|\hat{v}\|_{2, Q}^{2}\right) .
\end{aligned}
$$

Letting $h$ to 0 in (4.31), using (4.6) and the Korn inequality one obtains

$$
\left\|\tilde{v}_{z \tau \tau}\right\|_{0, Q}^{2} \leqq c\left(X_{2}+X_{3}\right)+\varepsilon\left(\|\hat{\eta}\|_{2, Q}^{2}+\|\hat{v}\|_{3, Q}^{2}\right) \text {. }
$$


Applying the operator $\delta_{h}^{l}, l<3$, to the third component of (4.22), multiplying by $\hat{V}_{n} \delta_{h}^{l} \tilde{\eta}$ and integrating over $Q$ yields

$$
\left\|\hat{V}_{n} \delta_{h}^{l} \tilde{\eta}\right\|_{0, Q}^{2} \leqq c\left\|\tilde{v}_{z \tau \tau}\right\|_{0, Q}^{2}+c d\left\|\tilde{v}_{z z \tau}\right\|_{0, Q}^{2}+\varepsilon\left(\|\hat{\eta}\|_{2, Q}^{2}+\|\hat{v}\|_{3, Q}^{2}\right)+c\left(X_{2}+X_{3}\right),
$$

where $d=\operatorname{diam} Q$ [see explanation after (4.18)]. Using that $\partial z_{i} / \partial y_{j}=\delta_{i j}+\nabla_{y_{j}} F_{i}$ and $\left.\nabla_{y} F\right|_{y=0}=0$ the first two terms in the right-hand side follow from

$$
\begin{aligned}
\left\|\delta_{h}^{l}(\hat{\Delta} \tilde{v}-\hat{\nabla} \widehat{\operatorname{div}} \tilde{v})\right\|_{0, Q} \leqq & \left\|(\hat{\Delta} \tilde{v}-\hat{\nabla} \widehat{\operatorname{div}} \tilde{v})_{\tau}\right\|_{0, Q} \\
\leqq & \left\|\Delta_{z} \tilde{v}_{\tau}-\nabla_{z} \operatorname{div}_{z} \tilde{v}_{\tau}\right\|_{0, Q} \\
& +P\left(\left\|z_{y}\right\|_{3, Q}\right)\left[|\hat{\nabla} \hat{F}|_{\infty, Q}\left\|\tilde{v}_{z z \tau}\right\|_{0, Q}+\|\tilde{v}\|_{2, Q}\right],
\end{aligned}
$$

where

and

$$
\left\|\Delta_{z} \tilde{v}_{\tau}-\nabla_{z} \operatorname{div}_{z} \tilde{v}_{\tau}\right\|_{0, Q} \leqq c\left\|\tilde{v}_{z \tau \tau}\right\|_{0, Q}, \quad|\hat{\nabla} \hat{F}|_{\infty, Q} \leqq c\|\hat{\nabla} \hat{F}\|_{3, Q} d
$$

$$
\left\|\hat{\nabla}_{n} \tilde{\eta}_{\tau}\right\|_{0, Q}^{2} \geqq\left\|\tilde{\eta}_{n \tau}\right\|_{0, Q}^{2}-c d\left\|\tilde{\eta}_{z \tau}\right\|_{2, Q}^{2} .
$$

To get this estimate we have used the following inequalities:

$$
\begin{aligned}
& \int_{Q} \delta_{h}^{l} \hat{\nabla}_{n}(\hat{\nabla} \cdot(\hat{w} \tilde{\eta})) \hat{\nabla}_{n} \delta_{h}^{l} \tilde{\eta} d z \leqq \varepsilon\left\|\hat{\nabla}_{n} \delta_{h}^{l} \tilde{\eta}\right\|_{0, Q}^{2}+c\|\hat{w}\|_{3, Q}\left\|\hat{\nabla}_{n} \delta_{h}^{l} \tilde{\eta}\right\|_{0, Q}^{2} \\
& +P\left(\left\|\hat{z}_{y}\right\|_{3, Q}\right)\|\hat{w}\|_{3, Q}\|\tilde{\eta}\|_{2, Q}^{2} \\
& \leqq \varepsilon\|\hat{\eta}\|_{2, Q}^{2}+c P\left(\left\|\hat{z}_{y}\right\|_{3, Q}\right)\|\hat{w}\|_{3, Q}^{2}\|\hat{\eta}\|_{2, Q}^{2},
\end{aligned}
$$

where the boundary term which appears in estimating the left-hand side vanishes in the same way as in (4.25), and

$$
\begin{aligned}
\int_{Q} \delta_{n}^{l} k_{3} \nabla_{n} \delta_{n}^{l} \tilde{\eta} d z \leqq & \varepsilon\left\|\hat{\nabla}_{n} \delta_{h}^{l} \tilde{\eta}\right\|_{0, Q}^{2} \\
& +P\left(\left\|\hat{z}_{y}\right\|_{3, Q}\right)\left(\|\hat{w}\|_{2, Q}^{2} \cdot\|\hat{\eta}\|_{2, Q}^{2}+\|\hat{v}\|_{2, Q}^{2}+\|\hat{\eta}\|_{1, Q}^{2}\right) .
\end{aligned}
$$

Letting $h$ to 0 in (4.35) we have

$$
\left\|\tilde{\eta}_{n \tau}\right\|_{0, Q}^{2} \leqq c\left\|\tilde{v}_{z \tau \tau}\right\|_{0, Q}^{2}+(c d+\varepsilon)\left(\|\hat{v}\|_{3, Q}^{2}+\|\hat{\eta}\|_{2, Q}^{2}+c\left(X_{2}+X_{3}\right) .\right.
$$

Equation (4.19) can be written in the form

$$
(\bar{\mu}+\bar{v}) \hat{\nabla} \widehat{\operatorname{div}} \tilde{v}=-\bar{\mu}(\hat{\nabla} \hat{\nabla} \tilde{v}-\hat{\nabla} \widehat{\operatorname{div}} \tilde{v})+p_{1} \hat{\nabla} \tilde{\eta}-\tilde{K}-k_{1} .
$$

Differentiating the third component of (4.37) with respect to $\tau$ yields

$$
\begin{aligned}
\left\|\hat{V}_{n} \widehat{\operatorname{div}} \tilde{v}_{\tau}\right\|_{0, Q}^{2} \leqq & \left\|\tilde{v}_{z \tau \tau}\right\|_{0, Q}^{2}+c d\left(\|\hat{v}\|_{3, Q}^{2}+\|\hat{\eta}\|_{2, Q}\right) \\
& +c\left\|\tilde{\eta}_{n \tau}\right\|_{0, Q}^{2}+c\left(X_{2}+X_{3}\right) .
\end{aligned}
$$

From (4.34), (4.36), and (4.38) one obtains

$$
\left\|\tilde{\eta}_{n \tau}\right\|_{0, Q}^{2}+\left\|(\widehat{\operatorname{div}} \tilde{v})_{n \tau}\right\|_{0, Q}^{2} \leqq(\varepsilon+c d)\left(\|\hat{\eta}\|_{2, Q}^{2}+\|\hat{v}\|_{3, Q}^{2}\right)+c\left(X_{2}+X_{3}\right) .
$$


Differentiating with respect to $\tau$ the Stokes problem

$$
\begin{gathered}
\bar{A}_{z} \tilde{v}+p_{1} \nabla_{z} \tilde{\eta}=\left(\bar{A}_{z}-\hat{\bar{A}}\right) \tilde{v}+\left(\nabla_{z}-\hat{\nabla}\right) \tilde{\eta}+\tilde{K}+k_{1}, \quad \text { in } Q \\
\operatorname{div}_{z} \tilde{v}=\operatorname{div}_{z} \tilde{v} \quad \text { in } Q, \\
\tilde{v} \bar{n}_{z}=\tilde{v} \bar{n}_{z}-\hat{v} \hat{\bar{n}} \text { on } \hat{S}, \\
\bar{\tau}_{z} T_{z}^{\prime}(\tilde{v}) \bar{n}_{z}=\bar{\tau}_{z} T_{z}^{\prime}(\tilde{v}) \bar{n}_{z}-\hat{\bar{\tau}} \hat{T}^{\prime}(\hat{v}) \hat{\bar{n}} \hat{\zeta} \quad \text { on } \hat{S} \\
\tilde{v} \cdot \bar{n}_{z}=0 \text { on } \partial Q \backslash \hat{S} \\
\bar{\tau}_{z} T_{z}^{\prime}(\tilde{v}) \bar{n}_{z}=0 \text { on } \partial Q \backslash \hat{S}
\end{gathered}
$$

where $\bar{n}_{z}=(0,0,1)$ and $\bar{\tau}_{z}^{1}=(1,0,0), \bar{\tau}_{z}^{2}=(0,1,0)$, and using Theorem 3.3 gives

$$
\begin{aligned}
\left\|\tilde{v}_{z z \tau}\right\|_{0, Q}^{2}+\left\|\tilde{\eta}_{z \tau}\right\|_{0, Q}^{2} \leqq & c\left\|\left(\operatorname{div}_{z} \tilde{v}\right)_{z \tau}\right\|_{0, Q}^{2}+c d\left(\|\hat{\eta}\|_{2, Q}^{2}\right. \\
& \left.+\|\hat{v}\|_{3, Q}^{2}\right)+c\left(X_{2}+X_{3}\right) .
\end{aligned}
$$

Applying the operator $\delta_{h}^{3}, h>0$, to the third component of (4.22), multiplying by $\hat{\nabla}_{n} \delta_{h}^{3} \tilde{\eta}$ and integrating over $Q$ one obtains

$$
\begin{aligned}
\left\|\hat{\nabla}_{n} \delta_{h}^{3} \tilde{\eta}\right\|_{0, Q} \leqq & c\left\|\tilde{v}_{z n \tau}\right\|_{0, Q}^{2}+c d\|\hat{v}\|_{3, Q}^{2} \\
& +\varepsilon\left(\|\hat{\eta}\|_{2, Q}^{2}+\|\hat{v}\|_{3, Q}^{2}\right)+c\left(X_{2}+X_{3}\right),
\end{aligned}
$$

where analogous considerations are carried out as in the case of inequality (4.35). Hence letting $h$ to 0 we have

$$
\left\|\tilde{\eta}_{n n}\right\|_{0, Q}^{2} \leqq c\left\|\tilde{v}_{z n \tau}\right\|_{0, Q}^{2}+(\varepsilon+c d)\left(\|\hat{\eta}\|_{2, Q}^{2}+\|\hat{v}\|_{3, Q}^{2}\right)+c\left(X_{2}+X_{3}\right) .
$$

Differentiating the third component of (4.37) with respect to $n$ shows that

$$
\begin{aligned}
\left\|(\widehat{\operatorname{div}} \tilde{v})_{n n}\right\|_{0, Q}^{2} \leqq & c\left\|\tilde{v}_{z n \tau}\right\|_{0, Q}^{2}+c d\|\hat{v}\|_{3, Q}^{2} \\
& +c\left\|\hat{\nabla}_{n} \tilde{\eta}_{n}\right\|_{0, Q}^{2}+c\left(X_{2}+X_{3}\right) .
\end{aligned}
$$

Now (4.43) and (4.44) imply

$$
\begin{gathered}
\left\|\tilde{\eta}_{n n}\right\|_{0, Q}^{2}+\left\|(\widehat{\operatorname{div}} \tilde{v})_{n n}\right\|_{0, Q}^{2} \leqq \\
c\left\|\tilde{v}_{z n \tau}\right\|_{0, Q}^{2}+(\varepsilon+c d)\left(\|\hat{\eta}\|_{2, Q}^{2}+\|\hat{v}\|_{3, Q}^{2}\right) \\
+c\left(X_{2}+X_{3}\right) .
\end{gathered}
$$

Finally, from (4.39), (4.41), and (4.45) we have

$$
\begin{aligned}
\left\|\tilde{v}_{z z \tau}\right\|_{0, Q}^{2} & +\left\|(\widehat{\operatorname{div}} \tilde{v})_{z z}\right\|_{0, Q}^{2}+\left\|\tilde{\eta}_{z z}\right\|_{0, Q}^{2} \\
& \leqq c(d+\varepsilon)\left(\|\hat{\eta}\|_{2, Q}^{2}+\|\hat{v}\|_{3, Q}^{2}\right)+c\left(X_{2}+X_{3}\right) .
\end{aligned}
$$

Going back to the old coordinates, summing over all neighbourhoods of the partition of unity and using Lemma 4.1 implies

$$
\|v\|_{2}^{2}+\|\eta\|_{2}^{2}+\left\|v_{x x \tau}\right\|_{0}^{2}+\left\|(\operatorname{div} v)_{x x}\right\|_{0}^{2} \leqq c(d+\varepsilon)\left(\|v\|_{3}^{2}+\|\eta\|_{2}^{2}\right)+c Y_{2},
$$

where $\tau$ signifies that near the boundary only the tangent derivatives appear. Using Theorem 3.2 to the Stokes problem (4.12) gives

$$
\|v\|_{3}^{2}+\|\eta\|_{2}^{2} \leqq c\left(\|\operatorname{div} v\|_{2}^{2}+\|K\|_{1}^{2}\right) \text {. }
$$


Hence from (4.47) and (4.48) for sufficiently small $d$ and $\varepsilon$ we get (4.15). From Lemma 4.2 we have

Theorem 4.3. Let $v, \eta$ be a solution to problem (4.1), (4.2), (4.4). Let (4.3) and (4.6) be satisfied. Let $A \equiv\|w\|_{3}$ be sufficiently small. Then

$$
\|v\|_{3}^{2}+\|\eta\|_{2}^{2} \leqq c_{1}\left(\|K\|_{1}^{2}+\|G\|_{2}^{2}\right) .
$$

Now we prove the existence of solutions to the linear problem (4.1), (4.2), (4.4) with conditions (4.3) and (4.6), where $w \in H^{3}(\Omega)$ is treated as a given function satisfying (4.5). We follow the method of [62].

First, we consider the case of $\xi / \mu$ large enough. Defining

$$
\pi \equiv \varrho p_{1} \eta / \mu-(\xi / \mu+1 / 3) \operatorname{div} v
$$

we transform the problem into two problems

$$
\begin{gathered}
-\Delta v+\nabla \pi=\varrho K / \mu \quad \text { in } \Omega, \\
\operatorname{div} v=(\xi / \mu+1 / 3)^{-1}\left(\varrho p_{1} \eta / \mu-\pi\right) \quad \text { in } \Omega, \\
\left.v \cdot \bar{n}\right|_{S}=0 \quad \text { on } S, \\
\left.\bar{\tau}_{k} T^{\prime}(v) \bar{n}\right|_{S}=0, \quad k=1,2, \quad \text { on } S,
\end{gathered}
$$

and

$$
\begin{gathered}
\bar{\varrho}(\xi / \mu+1 / 3)^{-1} \bar{\varrho} p_{1} \eta / \mu+\operatorname{div}(w \eta)=\bar{\varrho}(\xi / \mu+1 / 3)^{-1} \pi+G \text { in } \Omega \\
\int_{\Omega} \eta=0 .
\end{gathered}
$$

We solve (4.51), (4.52) by means of a fixed point theorem. Having determined $\left(\eta^{*}, \pi^{*}\right)$ on the right-hand side of $(4.51)_{2}$, we first get $(v, \pi)$ from the Stokes problem (4.51), and then $\eta$ from (4.52). A fixed point of the map

$$
\Phi:\left(\eta^{*}, \pi^{*}\right) \rightarrow(\eta, \pi)
$$

is a solution of (4.51), (4.52). Set

$$
\begin{aligned}
K_{1} & \equiv\left\{\left(\eta^{*}, \pi^{*}\right) \in H^{2}(\Omega) \times H^{2}(\Omega): \int_{\Omega} \eta^{*}\right. \\
& \left.=\int_{\Omega} \pi^{*}=0,\left\|\eta^{*}\right\|_{2}^{2} \leqq B,\left\|\pi^{*}\right\|_{2}^{2} \leqq B\right\} .
\end{aligned}
$$

Using Theorem 3.2 to the Stokes problem (4.51) (this problem is equivalent to the problem considered in Sect. 3 so the same results hold) we have (for the reader's convenience we repeat the considerations in [62])

$$
\begin{aligned}
\|v\|_{3}^{2}+\|\pi\|_{2}^{2} & \leqq c\left[\mu^{-2}\|K\|_{1}^{2}+(\xi / \mu+1 / 3)^{-2}\left(\frac{\bar{\varrho}^{2} p_{1}^{2}}{\mu^{2}}\left\|\eta^{*}\right\|_{2}^{2}+\left\|\pi^{*}\right\|_{2}^{2}\right)\right] \\
& \leqq c_{2}\left[\mu^{-2}\|K\|_{1}^{2}+(\xi / \mu+1 / 3)^{-2}\left(\varrho^{2} p_{1}^{2} \mu^{-2}+1\right) B\right] .
\end{aligned}
$$

In the case of a domain $\Omega$ obtained by rotation about the vector $B, K$ must satisfy condition (3.4). We have to underline that we used Theorem 3.2 in the case $S \in H^{7 / 2}$. 
By Lemma 2.6, if

$$
\|w\|_{3} \leqq c_{3} \varrho^{-2} p_{1}(\xi+\mu / 3)^{-1}
$$

where $c_{3}=c_{3}(\Omega)$ is determined by embedding theorems, for solutions to problem (4.52) one obtains the estimate

$$
\begin{aligned}
\|\eta\|_{2}^{2} \leqq & c\left[\mu^{2} \varrho^{-4} p_{1}^{-2}(\xi / \mu+1 / 3)^{2}\|G\|_{2}^{2}+\mu^{2} \varrho^{-2} p_{1}^{2}\|\pi\|_{2}^{2}\right] \\
\leqq & c_{4}\left[\varrho^{-4} p_{1}^{-2}(\xi+\mu / 3)^{2}\|G\|_{2}^{2}\right. \\
& \left.+\varrho^{-2} p_{1}^{-2}\left(\|K\|_{1}^{2}+(\xi / \mu+1 / 3)^{-2}\left(\varrho^{-2} p_{1}^{2}+1\right) B\right)\right] .
\end{aligned}
$$

Assuming that the right-hand sides of (4.55) and (4.57) are less than $B$, which may be satisfied if

$$
\max \left\{c_{2} \mu^{-2}\|K\|_{1}^{2}, c_{4} \bar{\varrho}^{-4} p_{1}^{-2}(\xi+\mu / 3)^{2}\|G\|_{2}^{2}+\varrho^{-2} p_{1}^{-2}\|K\|_{1}^{2}\right\}<B
$$

and if $\xi / \mu$ is large enough, we obtain $\|\pi\|_{2}^{2}<B,\|\eta\|_{2}^{2} \leqq B$. This means that $\Phi\left(K_{1}\right) \subset K_{1}$. The set $K_{1}$ is a convex compact subset of $Z \equiv H^{1}(\Omega) \times H^{1}(\Omega)$. Moreover, $\Phi: K_{1} \rightarrow K_{1}$ is continuous in the $Z$ topology. Hence by Schauder's theorem, $\Phi$ has a fixed point.

In this way we have proved the existence of solutions to (4.51), (4.52) for sufficiently large $\xi / \mu$ and small $\|w\|_{3}$.

To prove the existence of solutions of problem (4.51), (4.52) in the general case $(\xi / \mu$ arbitrary, $\xi \geqq 2 / 3 \mu)$ we use the same continuity method as in [62], applied to the following operators :

$$
\begin{aligned}
& \mu_{t}=(1-t) \mu_{0}+t \mu, \xi_{t}=(1-t) \xi_{0}+t \xi, t \in[0,1], \\
& L_{t}(v, \eta) \equiv\left(-\mu_{t} \Delta v-\left(\xi_{t}+1 / 3 \mu_{t}\right) \nabla \operatorname{div} v+\varrho p_{1} \nabla \eta, \varrho \operatorname{div} v+\operatorname{div}(w \eta)\right)=(K, G), \\
& X \equiv\left\{(v, \eta) \in H^{3}(\Omega) \times H^{2}(\Omega),\left.v \cdot \bar{n}\right|_{s}=0,\left.\bar{\tau} T^{\prime}(v) \bar{n}\right|_{s}=0, \int_{\Omega} \eta=0\right\}, \\
& Y \equiv\left\{(K, G) \in H^{1}(\Omega) \times H^{2}(\Omega), \int_{\Omega} G d x=0\right\},
\end{aligned}
$$

where $\xi_{0} / \mu_{0}$ is large enough and such that (4.56) is satisfied (see the previous part of the proof). It can be easily proved that the set $T \equiv\{t \in[0,1]$ : for each $(K, G) \in Y$ there exists a unique solution

$$
\left.(v, \eta) \in X \quad \text { of } \quad L_{t}(v, \eta)=(K, G)\right\}
$$

is nonempty and $T \equiv[0,1]$ (see [62]).

Hence we have proved

Theorem 4.4. Let $K \in H^{1}(\Omega), G \in H^{2}(\Omega), \xi>(2 / 3) \mu, S \in H^{7 / 2}, w \in H^{3}(\Omega)$ and suppose $A=\|w\|_{3}$ is sufficiently small (see (4.56) and assumptions of Theorem 4.3). Then there exists a solution $(v, \eta) \in H^{3}(\Omega) \times H^{2}(\Omega)$ of problem (4.51), (4.52) such that

$$
\|v\|_{3}+\|\eta\|_{2} \leqq c_{5}\left(\|K\|_{1}+\|G\|_{2}\right) \leqq A
$$


Now we are in a position to prove the existence of solutions to the nonlinear problem (1.1). Set

$K_{2}=\left\{(v, \eta) \in H^{3}(\Omega) \times H^{2}(\Omega),\left.v \cdot \bar{n}\right|_{S}=0,\left.\bar{\tau} \cdot T^{\prime}(v) \bar{n}\right|_{S}=0, \int_{\Omega} \eta=0,\|v\|_{3}+\|\eta\|_{2} \leqq D\right\}$,

where $D$ will be chosen later. To use Theorem 4.4 we must require that $D \leqq A$. Setting

$$
K=\left(p_{1}-\frac{p_{\varrho}(\bar{\varrho}+\sigma)}{\bar{\varrho}+\sigma}\right) \nabla \sigma-w \cdot \nabla w+\frac{\sigma}{\bar{\varrho}+\sigma} \bar{A} w+f, \quad G=0
$$

in (4.51) and (4.52), Theorem 4.4 implies the map

From (4.59) and (4.60) we have

$$
\Psi:(w, \sigma) \rightarrow(v, \eta) \text {. }
$$

$$
\begin{aligned}
\|v\|_{3}+\|\eta\|_{2} & \leqq c_{5}\|K\|_{1} \leqq c_{6}\left(\|\sigma\|_{2}^{2}+\|\sigma\|_{2}^{3}+\|w\|_{3}^{2}+\|\sigma\|_{2}\|w\|_{3}+\|\sigma\|_{2}^{2}\|w\|_{3}+\|f\|_{1}\right) \\
& \leqq c_{7}\left[D^{2}(1+D)+\|f\|_{1}\right] .
\end{aligned}
$$

Choosing $D$ and $\|f\|_{1}$ so small that the right-hand side of (4.62) is less than $D$ we get $\Psi\left(K_{2}\right) \subset K_{2}$. On the other hand, $K_{2}$ is convex and compact in $Z_{1} \equiv H^{2}(\Omega) \times H^{1}(\Omega)$ and $\Psi$ is continuous in the topology of $Z_{1}$. Hence by Schauder's theorem, $\Psi$ has a fixed point which is a solution to (1.1). Therefore we have proved

Theorem 4.5. Let $f \in H^{1}(\Omega)$ with $\|f\|_{1}$ sufficiently small, $S \in H^{7 / 2}$. Assume (4.6). Then there exists a solution $(v, \eta) \in H^{3}(\Omega) \times H^{2}(\Omega)$ to problem (1.1) and

$$
\|v\|_{3}+\|\eta\|_{2} \leqq c\|f\|_{1} \text {. }
$$

Remark 4.6. Suppose a bounded domain $\Omega$ is obtained by rotation about the vector $B$ and satisfies symmetry conditions (5.1). Suppose $f$ satisfies symmetry conditions (5.4) and $\|f\|_{1}$ is sufficiently small. Then there exists a symmetric solution to problem (1.1) determined by Theorem 4.5 and satisfying (5.6).

\section{Variation of a Free Boundary}

Let $\Omega^{(1)}$ and $\Omega^{(2)}$ be two domains given by

$$
\Omega^{(i)}=\left\{x: r<\zeta^{(i)}(\theta)\right\}, \quad i=1,2,
$$

where $\zeta^{(i)}(\theta)=R_{0}+R^{(i)}(\theta), R_{0}=$ const and $R^{(i)}(\theta)$ satisfies the symmetry conditions

$$
\frac{d^{l} R^{(i)}}{d \theta^{l}}(\pi / 2+\theta)=(-1)^{l} \frac{d^{l} R^{(i)}}{d \theta^{l}}(\pi / 2-\theta), \quad l=0,1,2,3 .
$$

Assume that $R^{(i)} \in H^{7 / 2}(0, \pi)$. Using the implicit function theorem one can show (we omit the simple but cumbersome calculations) that the surfaces $\Gamma^{(i)}=\partial \Omega^{(i)}$ are then of class $H^{7 / 2}$. Suppose additionally that

$$
\left\|R^{(1)}-R^{(2)}\right\|_{7 / 2,(0, \pi)}<\varepsilon_{0}, \quad\left\|R^{(i)}\right\|_{7 / 2,(0, \pi)} \leqq R_{0} / 2
$$

with sufficiently small $\varepsilon_{0}$. 
Consider in $\Omega^{(1)}$ and $\Omega^{(2)}$ the boundary value problem (1.1) with the additional condition

$$
\int_{\Omega^{(i)}} \varrho^{(i)}(x) d x=M .
$$

Assume that the right-hand side of (1.1), $f$, has norm $\|f\|_{c^{2}\left(\Omega^{(i)}\right)}$ sufficiently small and satisfies the symmetry conditions: $\left(f_{r}, f_{\varphi}, f_{\theta}\right)=\left(f_{r}, 0, f_{\theta}\right)$, the functions $f_{r}, f_{\theta}$ depend only on $(r, \theta)$ and

$$
\begin{aligned}
& \frac{\partial^{l} f_{r}}{\partial \theta^{l}}(r, \pi / 2+\theta)=(-1)^{l} \frac{\partial^{l} f_{r}}{\partial \theta^{l}}(r, \pi / 2-\theta), \\
& \frac{\partial^{l} f_{\theta}}{\partial \theta^{l}}(r, \pi / 2+\theta)=(-1)^{l+1} \frac{\partial^{l} f_{\theta}}{\partial \theta^{l}}(r, \pi / 2-\theta), \quad l=0,1 .
\end{aligned}
$$

Then the necessary and sufficient condition for the solvability of problem (1.1) is satisfied and by virtue of Theorem 4.5 and Remark 4.6 there exists a solution $\left(v^{(i)}, \varrho^{(i)}\right)$ of $(1.1)$ in $\Omega^{(i)}$ such that $\varrho^{(i)}$ admits the representation

$$
\varrho^{(i)}(x)=\bar{\varrho}^{(i)}+\eta^{(i)}(x),
$$

where $\bar{\varrho}^{(i)}=M / \Omega^{(i)}|,| \Omega^{(i)} \mid=\operatorname{vol} \Omega^{(i)}=\frac{2 \pi}{3} \int_{0}^{\pi}\left(R_{0}+R^{(i)}(\theta)\right)^{3} \sin \theta d \theta$,

$$
\int_{\Omega^{(i)}} \eta^{(i)}(x) d x=0
$$

and $\left(v^{(i)}, \eta^{(i)}\right)$ satisfies the following symmetry conditions: $v^{(i)}, \eta^{(i)}$ depend only on $r, \theta,\left(v_{r}^{(i)}, v_{\varphi}^{(i)}, v_{\theta}^{(i)}\right)=\left(v_{r}^{(i)}, 0, v_{\theta}^{(i)}\right)$,

$$
\begin{gathered}
\frac{\partial^{l} v_{r}^{(i)}}{\partial \theta^{l}}(r, \pi / 2+\theta)=(-1)^{l} \frac{\partial^{l} v_{r}^{(i)}}{\partial \theta^{l}}(r, \pi / 2-\theta), \\
\frac{\partial^{l} v_{\theta}^{(i)}}{\partial \theta^{l}}(r, \pi / 2+\theta)=(-1)^{l+1} \frac{\partial v_{\theta}^{(i)}}{\partial \theta^{l}}(r, \pi / 2-\theta), \quad l=0,1,2,3, \\
\frac{\partial^{l} \eta^{(i)}}{\partial \theta^{l}}(r, \pi / 2+\theta)=(-1)^{l} \frac{\partial^{l} \eta^{(i)}}{\partial \theta^{l}}(r, \pi / 2-\theta), \quad l=0,1,2 .
\end{gathered}
$$

Moreover, $\left(v^{(i)}, \eta^{(i)}\right)$ obeys the estimates

$$
\left\|v^{(i)}\right\|_{3, \Omega^{(i)}}+\left\|\eta^{(i)}\right\|_{2, \Omega^{(i)}} \leqq c\|f\|_{1, \Omega^{(i)}}, \quad i=1,2 .
$$

Note that for sufficiently small $\varepsilon_{0}$ in (5.2) the constant in (5.7) can be chosen the same for all $R^{(i)}$ satisfying (5.2).

We now compare $\left(v^{(1)}, \varrho^{(1)}\right)$ with $\left(v^{(2)}, \varrho^{(2)}\right)$. For this purpose we construct a transformation which maps $\Omega^{(1)}$ onto $\Omega^{(2)}$. Let $x=\left(x_{1}, x_{2}, x_{3}\right)$ and $(r, \varphi, \theta)$ be Cartesian and spherical coordinates in $\Omega^{(1)}$ and let $y=\left(y_{1}, y_{2}, y_{3}\right)$ and $(\hat{r}, \varphi, \theta)$ be the same coordinates in $\Omega^{(2)}$. Introduce the transformation

$$
\hat{r}=r+\lambda\left(\frac{r}{R_{0}+R^{(1)}(\theta)}\right) \omega(r, \theta),
$$


where $\lambda(t)$ is a smooth cut-off function with $\lambda(t)=0$ in a neighbourhood of $t=0$ and $\lambda(t)=1$ for $t \geqq 1 / 4$, and $\omega \in H^{4}\left(\Omega^{(1)}\right)$ satisfies the boundary condition

and the inequality

$$
\left.\omega(r, \theta)\right|_{r=R_{0}+R^{(1)}(\theta)}=R^{(2)}(\theta)-R^{(1)}(\theta),
$$

$$
\|\omega\|_{4, \Omega^{(1)}} \leqq c\left\|R^{(1)}-R^{(2)}\right\|_{7 / 2,(0, \pi)} .
$$

One can easily show that the transformation (5.8) maps $\Gamma^{(1)}$ onto $\Gamma^{(2)}$ and for sufficiently small $\varepsilon_{0}$ its jacobian is bounded away from zero. Hence (5.8) defines a one-to-one mapping $y=\Phi x$ of $\Omega^{(1)}$ onto $\Omega^{(2)}$. From (5.8)-(5.10) one gets the estimate

$$
\left\|x-\Phi^{-1} y\right\|_{4, \Omega^{(1)}}+\|y-\Phi x\|_{4, \Omega^{(2)}} \leqq c\left\|R^{(2)}-R^{(1)}\right\|_{7 / 2,(0, \pi)} .
$$

Let us rewrite problem (1.1) for $v^{(1)}, \varrho^{(1)}$ in the new coordinates $y$ :

$$
\begin{gathered}
-\mu \hat{\nabla}^{2} \hat{v}^{(1)}-v \hat{\nabla} \widehat{\operatorname{div}} \hat{v}^{(1)}+\left(\bar{\varrho}^{(1)}+\hat{\eta}^{(1)}\right)\left(\hat{v}^{(1)} \hat{\nabla}\right) \hat{v}^{(1)}+\hat{\nabla} p\left(\bar{\varrho}^{(1)}+\hat{\eta}^{(1)}\right) \\
=\left(\varrho^{(1)}+\hat{\eta}^{(1)}\right) \hat{f}, \hat{\nabla} \cdot\left[\left(\varrho^{(1)}+\hat{\eta}^{(1)}\right) \hat{v}^{(1)}\right]=0,\left.\quad \hat{v}^{(1)} \cdot \hat{n}\right|_{\Gamma^{(2)}}=0, \\
\hat{T}^{\prime}\left(\hat{v}^{(1)}\right) \hat{n}-\left.\left(\hat{n} \cdot \hat{T}^{\prime}\left(\hat{v}^{(1)}\right) \hat{n}\right) \hat{n}\right|_{\Gamma^{(2)}}=0,
\end{gathered}
$$

where $\quad \hat{v}^{(1)}(y)=v^{(1)}\left(\Phi^{-1} y\right), \quad \hat{\eta}^{(1)}(y)=\eta^{(1)}\left(\Phi^{-1} y\right), \quad \hat{f}(y)=f\left(\Phi^{-1} y\right), \quad \hat{\nabla}=A(y) \nabla$, $\hat{n}=\frac{A \bar{n}}{|A \bar{n}|}=B \bar{n}, A$ and $\hat{T}^{\prime}$ are matrices with elements

$$
a_{i j}(y)=\left.\frac{\partial x_{j}}{\partial x_{i}}\right|_{y=\Phi^{-1} x}, \quad \hat{T}_{i j}^{\prime}=\sum_{m=1}^{3}\left(a_{i m} \frac{\partial v_{j}^{(1)}}{\partial x_{m}}+a_{j m} \frac{\partial v_{i}^{(1)}}{\partial x_{m}}\right), \quad i, j=1,2,3 .
$$

Subtracting equations (1.1) for $\left(v^{(2)}, \varrho^{(2)}\right)$ from system (5.12) we get the following boundary value problem for the differences $u(y)=\hat{v}^{(1)}(y)-v^{(2)}(y)$, $Q(y)=\hat{\eta}^{(1)}(y)-\eta^{(2)}(y)$ :

$$
\begin{gathered}
-\mu \nabla^{2} u-v \nabla \operatorname{div} u+p^{\prime}\left(\bar{\varrho}^{(1)}\right) \nabla Q=F_{1}+F_{2}+F_{3}, \\
\bar{\varrho}^{(1)} \nabla \cdot u=G_{1}+G_{2}, \\
\left.u \cdot \bar{n}\right|_{\Gamma^{(2)}}=b, \quad T^{\prime}(u) \bar{n}-\left.\bar{n}\left(\bar{n} \cdot T^{\prime}(u) \bar{n}\right)\right|_{\Gamma^{(2)}}=d
\end{gathered}
$$

with

$$
\begin{gathered}
F_{1}=\mu\left(\hat{\nabla}^{2}-\nabla^{2}\right) \hat{v}^{(1)}+v(\hat{\nabla} \widehat{\operatorname{div}}-\nabla \operatorname{div}) \hat{v}^{(1)}-(\hat{\nabla}-\nabla) p\left(\bar{\varrho}^{(1)}+\hat{\eta}^{(1)}\right)-\left(\bar{\varrho}^{(1)}+\hat{\eta}^{(1)}\right) \\
\cdot\left((\hat{\nabla}-\nabla) \cdot \hat{v}^{(1)}\right) \hat{v}^{(1)}-\left(\bar{\varrho}^{(1)}-\bar{\varrho}^{(2)}\right)\left(\nabla \cdot v^{(2)}\right) v^{(2)}+\bar{\varrho}^{(1)}(\hat{f}-f) \\
+\hat{\eta}^{(1)}(\hat{f}-f)+\left(\bar{\varrho}^{(1)}-\bar{\varrho}^{(2)}\right) f ; \\
F_{2}=Q f-\left(\bar{\varrho}^{(1)}+\hat{\eta}^{(1)}\right)(\nabla \cdot u) \hat{v}^{(1)}-\left(\bar{\varrho}^{(1)}+\hat{\eta}^{(1)}\right)\left(\nabla \cdot v^{(2)}\right) u-Q\left(\nabla \cdot v^{(2)}\right) v^{(2)} ; \\
F_{3}=-\left[\left(p^{\prime}\left(\bar{\varrho}^{(1)}+\hat{\eta}^{(1)}\right)-p^{\prime}\left(\bar{\varrho}^{(2)}+\eta^{(2)}\right)\right) \nabla \eta^{(2)}+\left(p^{\prime}\left(\bar{\varrho}^{(1)}+\hat{\eta}^{(1)}\right)-p^{\prime}\left(\bar{\varrho}^{(1)}\right)\right) \nabla Q\right] ; \\
G_{1}=(\nabla-\hat{\nabla}) \cdot\left(\left(\bar{\varrho}^{(1)}+\hat{\eta}^{(1)}\right) \hat{v}^{(1)}\right)+\left(\bar{\varrho}^{(2)}-\bar{\varrho}^{(1)}\right) \nabla \cdot v^{(2)} ; \\
G_{2}=-\nabla \cdot\left(\hat{\eta}^{(1)} u\right)-\nabla \cdot\left(Q v^{(2)}\right) ; \\
b=\left.\hat{v}^{(1)} \cdot(\hat{n}-\bar{n})\right|_{\Gamma^{(2)}}=\left.\hat{v}^{(1)} \cdot(B-I) \bar{n}\right|_{\Gamma^{(2)}} ; \quad d=\left\{\left[\hat{T}^{\prime}\left(\hat{v}^{(1)}\right)-T^{\prime}\left(v^{(2)}\right)\right] \bar{n}+\hat{T}^{\prime}\left(\hat{v}^{(1)}\right)\right. \\
\left.\cdot(\hat{n}-\bar{n})+\bar{n}\left[\bar{n} \cdot\left(\hat{T}^{\prime}\left(\hat{v}^{(1)}\right)-T^{\prime}\left(\hat{v}^{(1)}\right)\right) \bar{n}\right]+\left[\hat{n}\left(\hat{n} \cdot T^{\prime}\left(\hat{v}^{(1)}\right) \hat{n}\right)-\bar{n}\left(\bar{n} \cdot T^{\prime}\left(\hat{v}^{(1)}\right) \bar{n}\right)\right]\right\}\left.\right|_{\Gamma^{(2)}} .
\end{gathered}
$$


Using inequalities (5.7), $i=1,2,(5.11)$ and the formulas

$$
\bar{\varrho}^{(i)}=3 M /\left(2 \pi \int_{0}^{\pi}\left(R_{0}+R^{(i)}(\theta)\right)^{3} \sin \theta d \theta\right)
$$

one gets the estimates

$$
\begin{gathered}
\left\|F_{1}\right\|_{1, \Omega^{(2)}}+\left\|G_{1}\right\|_{2, \Omega^{(2)}} \leqq c(|f|)\left\|R^{(1)}-R^{(2)}\right\|_{7 / 2,(0, \pi)}, \\
\left\|F_{2}\right\|_{1, \Omega^{(2)}}+\left\|G_{2}\right\|_{2, \Omega^{(2)}} \leqq c(|f|)\left(\|Q\|_{2, \Omega^{(2)}}+\|u\|_{3, \Omega^{(2)}}\right) \\
\left\|F_{3}\right\|_{1, \Omega^{(2)}}+\|b\|_{7 / 2, \Gamma^{(2)}}+\|d\|_{5 / 2, \Gamma^{(2)}} \\
\leqq c(|f|)\left(\|Q\|_{2, \Omega^{(2)}}+\|u\|_{3, \Omega^{(2)}}+\left\|R^{(1)}-R^{(2)}\right\|_{7 / 2,(0, \pi)}\right) .
\end{gathered}
$$

Here $|f|=\|f\|_{c^{2}\left(\Omega^{(2)}\right)}$, and the constant $c(|f|)$ is small for small $|f|$. Note that in deriving (5.14) we also use imbedding theorems, the properties of the function $p$ and the estimate

$$
\begin{aligned}
\left\|f\left(\Phi^{-1}(y)\right)-f(y)\right\|_{c^{1}\left(\Omega^{(2)}\right)} & \leqq c\|f\|_{c^{2}\left(\Omega^{(2)}\right)}\left|y-\Phi^{-1} y\right|_{4, \Omega^{(2)}} \\
& \leqq c|f|\left\|R^{(1)}-R^{(2)}\right\|_{7 / 2,(0, \pi)} .
\end{aligned}
$$

One easily verifies that the right-hand sides of system (5.13) satisfy condition (3.14). Hence we can get an estimate for $u, Q$ using Theorem $3.3(u, Q$ is a solution of the Stokes system). Since (see (5.5))

$$
\begin{aligned}
\left|\int_{\Omega^{(2)}} Q(y) d y\right| & =\left|\int_{\Omega^{(2)}} \eta^{(1)}\left(\Phi^{-1}(y)\right) d y\right| \\
& =\left|\int_{\Omega^{(2)}} \hat{\eta}^{(1)}(y)\left[\operatorname{det} A^{-1}-1\right] d y\right| \leqq c(|f|)\left\|R^{(1)}-R^{(2)}\right\|_{7 / 2,(0, \pi)}
\end{aligned}
$$

inequalities (3.15) and (5.14) yield

$$
\|u\|_{3, \Omega^{(2)}}+\|Q\|_{2, \Omega^{(2)}} \leqq c(|f|)\left(\|u\|_{3, \Omega^{(2)}}+\|Q\|_{2, Q^{(2)}}\right)+c(|f|)\left\|R^{(1)}-R^{(2)}\right\|_{7 / 2,(0, \pi)} .
$$

Hence for sufficiently small $|f|$,

$$
\|u\|_{3, \Omega^{(2)}}+\|Q\|_{2, \Omega^{(2)}} \leqq c(|f|)\left\|R^{(1)}-R^{(2)}\right\|_{7 / 2,(0, \pi)} .
$$

Consider the functions $\Psi^{(i)}(x)=\left.\left(-p\left(\varrho^{(i)}\right)+\bar{n} \cdot T^{\prime}\left(v^{(i)}\right) \bar{n}\right)\right|_{\Gamma^{(i)}}$. Since the solutions $\left(v^{(i)}, \varrho^{(i)}\right)$ satisfy the symmetry conditions $(5.6), \Psi^{(i)}(x)$ is a function of $\theta$ only and satisfies conditions (5.1). Therefore, we have proved the following

Theorem 5.1. The functions $\Psi^{(i)}(\theta)=\left.\left(\bar{n} \cdot T\left(v^{(i)}\right) \bar{n}-p\left(\varrho^{(i)}\right)\right)\right|_{r=R+R_{0}^{(i)}(\theta)}$ satisfy the symmetry conditions (5.6) and obey the estimate

$$
\left\|\Psi^{(1)}-\Psi^{(2)}\right\|_{3 / 2,(0, \pi)} \leqq c(|f|)\left\|R^{(1)}-R^{(2)}\right\|_{7 / 2,(0, \pi)} .
$$

(5.16) follows from (5.15). 


\section{Existence of Solutions}

To prove the existence for the original problem (1.1), (1.5), (1.6) we shall use a method of successive approximations. Before describing this method we transform Eq. (1.5). Assume that the free boundary $\Gamma$ is specified by $\Gamma=\left\{x: r=R_{0}+R(\varphi, \theta)\right\}$. We can write the mean curvature operator in spherical coordinates $(r, \varphi, \theta)$ for the function $\zeta(\varphi, \theta)=R_{0}+R(\varphi, \theta)$ as follows [22]:

$$
H(x)=L \zeta=-\frac{1}{\sin \theta}\left[\frac{\partial}{\partial \varphi}\left(h^{-1} \frac{\partial}{\partial \varphi} \zeta\right)+\frac{\partial}{\partial \theta}\left(h^{-1} \sin ^{2} \theta \frac{\partial}{\partial \theta} \zeta\right)\right]+2 \sin \theta h^{-1}
$$

where $h=\left(\zeta^{2} \sin ^{2} \theta+(\partial \zeta / \partial \varphi)^{2}+(\partial \zeta / \partial \theta)^{2} \sin ^{2} \theta\right)^{1 / 2}$. Linearizing the right-hand side of (6.1) one gets

$$
L \zeta=2 / R_{0}-1 / R_{0}^{2}\left(\Delta^{*}+2\right)\left(R-\Phi^{*}(R),\right.
$$

where $\Delta^{*}=1 / \sin \theta\left[\partial / \partial \varphi\left((\sin \theta)^{-1} \partial / \partial \varphi\right)+\partial / \partial \theta(\sin \theta \partial / \partial \theta)\right]$ is the Laplace-Beltrami operator on the sphere $S^{1}$ and

$$
\begin{aligned}
& \Phi^{*}(R)= R /\left(\zeta R_{0} \sin \theta\right)\left[\partial / \partial \varphi\left(h^{-1} \partial R / \partial \varphi\right)+\partial / \partial \theta\left(h^{-1} \sin ^{2} \theta \partial R / \partial \theta\right)\right] \\
&+\left.2 \sin \theta R^{2} \int_{0}^{1}(1-\tau) L_{1}^{2} \Psi(x, y, z)\right|_{\begin{array}{l}
x=R_{0}+\tau R \\
y=\tau \partial R / \partial \theta \\
z=\tau \partial R / \partial \varphi
\end{array}} \partial d \tau \\
&-1 /\left(R_{0} \sin \theta\right)\left\{\partial / \partial \varphi\left[\left.R(\partial R / \partial \varphi) \int_{0}^{1} L_{1} \Psi(x, y, z)\right|_{\substack{x=R_{0}+\tau R \\
y=\tau \partial R / \partial \theta \\
z=\tau \partial R / \partial \varphi}} d \tau\right]\right. \\
&+\partial / \partial \theta\left[\left.\sin ^{2} \theta R(\partial R / \partial \theta) \int_{0}^{1} L_{1} \Psi(x, y, z)\right|_{\begin{array}{l}
x=R_{0}+\tau R \\
y=\tau \partial R / \partial \theta \\
z=\tau \partial R / \partial \varphi
\end{array}} d \tau,\right. \\
& L_{1}=\partial / \partial x+\partial / \partial y+\partial / \partial z, \quad \Psi(x, y, z)=\left(\left(x^{2}+y^{2}\right) \sin ^{2} \theta+z^{2}\right)^{-1 / 2} .
\end{aligned}
$$

Now we consider in detail the properties of the operator $\left(\Delta^{*}+2\right)$, i.e. of the equation

$$
\left(\Delta^{*}+2\right) Z=F \text {. }
$$

Theorem 6.1. For any $F \in H^{m}\left(S^{1}\right)$ satisfying

$$
\int_{S^{1}} F(\varphi, \theta) \cos \theta d S^{1}=0,
$$

Eq. (6.3) has a unique solution $Z \in H^{m+2}\left(S^{1}\right)$ such that

$$
\int_{S^{1}} Z(\varphi, \theta) \cos \theta d S^{1}=0
$$

and we have the estimate

$$
\|Z\|_{m+2, S^{1}} \leqq c\|F\|_{m, S^{1}} .
$$

Proof. It is well known that the Laplace-Beltrami operator $\Delta^{*}$ is an elliptic self adjoint operator and hence (see e.g. [42, Chap. I, Sect. 8.2]) $\Delta^{*}$ has the Fredholm 
property in the scale of Sobolev spaces. Moreover, $\lambda=2$ is an eigenvalue of $\Delta^{*}$ with eigenfunction $U=\cos \theta$ (see e.g. [42, Chap. III, Sect. 22.2]). This implies the theorem.

Theorem 6.2. Let $F$ be a function of $\theta$ only, $F \in H^{3 / 2}(0, \pi)$, which satisfies the symmetry conditions

$$
\left(d^{l} F / d \theta^{l}\right)(\pi / 2+\theta)=(-1)^{l}\left(d^{l} F / d \theta^{l}\right)(\pi / 2-\theta), \quad l=0,1 .
$$

Then there exists a unique solution $Z \in H^{7 / 2}(0, \pi)$ of Eq. (6.3) also depending on $\theta$ only and such that

and

$$
\left(d^{l} Z / d \theta^{l}\right)(\pi / 2+\theta)=(-1)^{l}\left(d^{l} Z / d \theta^{l}\right)(\pi / 2-\theta), \quad l=0,1,2,3,
$$

$$
\|Z\|_{7 / 2,(0, \pi)} \leqq c\|F\|_{3 / 2,(0, \pi)} .
$$

Proof. For a function $Z$ independent of $\varphi$ the operator $\left(\Delta^{*}+2\right) Z$ takes the form

$$
\left(\Delta^{*}+2\right) Z=1 / \sin \theta(\partial / \partial \theta(\sin \theta(\partial Z / \partial \theta(\theta)))+2 Z(\theta) .
$$

Hence it is sufficient to solve the ordinary differential equation

$$
1 / \sin \theta d / d \theta(\sin \theta(d Z / d \theta)(\theta))+2 Z(\theta)=F(\theta), \quad \theta \in(0, \pi) .
$$

Introducing the variable $\eta=\cos \theta$ gives

$$
d / d \eta\left[\left(1-\eta^{2}\right)(d \hat{Z} / d \eta)(\eta)\right]+2 \hat{Z}(\eta)=\hat{F}(\eta), \quad \eta \in(-1.1),
$$

where $\hat{Z}(\eta)=Z(\arccos \eta), \hat{F}(\eta)=F(\arccos \eta)$. The homogeneous equation corresponding to $(6.11)$ has two linearly independent solutions $\hat{Z}_{1}(\eta)=\eta$ and $\hat{Z}_{2}(\eta)=1-1 / 2 \eta \ln ((1+\eta) /(1-\eta))$. The solution of the nonhomogeneous problem (6.11) satisfying (6.8) can be written in the form

$$
\hat{Z}(\eta)= \begin{cases}\hat{Z}_{1}(\eta) \int_{-1}^{\eta} \hat{Z}_{2}(\xi) \hat{F}(\xi) d \xi+\hat{Z}_{2}(\eta) \int_{\eta}^{1} \hat{Z}_{1}(\xi) \hat{F}(\xi) d \xi+\kappa_{0} \hat{Z}_{1}(\eta), & 0 \leqq \eta \leqq 1, \\ -\hat{Z}_{2}(\eta) \int_{-1}^{\eta} \hat{Z}_{1}(\xi) \hat{F}(\xi) d \xi-\hat{Z}_{1}(\eta) \int_{\eta}^{1} \hat{Z}_{2}(\xi) \hat{F}(\xi) d \xi-\kappa_{0} \hat{Z}_{1}(\eta), & -1 \leqq \eta \leqq 0,\end{cases}
$$

where $\kappa_{0}=-\int_{-1}^{0} \hat{Z}_{2}(\xi) \hat{F}(\xi) d \xi$. Using (6.7) one can easily calculate that

$$
\hat{Z}(-\eta)=\hat{Z}(\eta), \quad(d \hat{Z} / d \eta)(-\eta)=-(d \hat{Z} / d \eta)(\eta), \quad(d \hat{Z} / d \eta)(0)=0 .
$$

Let us estimate the norm

$$
\langle\hat{Z}\rangle_{1}=\left(\int_{-1}^{1} \hat{Z}(\eta)^{2} d \eta+\int_{-1}^{1}\left(1-\eta^{2}\right)|(d \hat{Z} / d \eta)(\eta)|^{2} d \eta\right)^{1 / 2},
$$

which is equivalent to $\|Z\|_{1, s^{1}}$. Since $\ln ((1+\eta) /(1-\eta)) \in L_{2}(-1,1)$ one easily gets the estimate

$$
\int_{-1}^{1}|\hat{Z}(\eta)|^{2} d \eta \leqq c\left(\int_{-1}^{1} \hat{F}(\eta)^{2} d \eta+\kappa_{0}^{2}\right) \leqq c \int_{-1}^{1} \hat{F}(\eta)^{2} d \eta
$$


From (6.12) we calculate

$$
\frac{d}{d \eta} \hat{Z}(\eta)= \begin{cases}\int_{-1}^{\eta} \hat{Z}_{2}(\xi) \hat{F}(\xi) d \xi+d / d \eta \hat{Z}_{2}(\eta) \int_{\eta}^{1} \xi \hat{F}(\xi) d \xi+\kappa_{0}, & 0 \leqq \eta \leqq 1 \\ -d \hat{Z}_{2} / d \eta(\eta) \int_{-1}^{\eta} \xi \hat{F}(\xi) d \xi-\int_{\eta}^{1} \hat{Z}_{2}(\xi) \hat{F}(\xi) d \xi-\kappa_{0}, & -1 \leqq \eta \leqq 0\end{cases}
$$

Since $\left|(d / d \eta) \hat{Z}_{2}(\eta)\right| \leqq c\left(1-\eta^{2}\right)^{-1}, \eta \in(-1,1)$, we have

$$
\begin{aligned}
\int_{-1}^{1}(1 & \left.-\eta^{2}\right)|(d / d \eta) \hat{Z}(\eta)|^{2} d \eta \\
& \leqq c\left(\kappa_{0}^{2}+\int_{0}^{1}\left(1-\eta^{2}\right)\left(\int_{-1}^{\eta} \hat{Z}_{2}(\xi) \hat{F}(\xi) d \xi\right)^{2} d \eta+\int_{0}^{1}\left(1-\eta^{2}\right)^{-1}\left(\int_{\eta}^{1} \xi \hat{F}(\xi) d \xi\right)^{2} d \eta\right) \\
& \leqq c\left(\int_{-1}^{1} \hat{F}(\xi)^{2} d \xi+\int_{0}^{1}\left(1-\eta^{2}\right)^{-1}\left(\int_{\eta}^{1} \xi d \xi\right) d \eta \int_{-1}^{1} \hat{F}(\xi)^{2} d \xi\right) \\
& \leqq c\left(\int_{-1}^{1} \hat{F}(\eta)^{2} d \eta\right) .
\end{aligned}
$$

Therefore $Z(\theta)=\hat{Z}(\cos \theta) \in H^{1}\left(S^{1}\right)$.

Now we prove that $\hat{Z}(\eta)$ is a unique solution of problem (6.11) belonging to $H^{1}\left(S^{1}\right)$ and satisfying (6.13). Indeed, the general solution of the homogeneous problem corresponding to $(6.11)$ is

$$
\hat{Z}_{h}(\eta)=c_{1} \hat{Z}_{1}(\eta)+c_{2} \hat{Z}_{2}(\eta)
$$

Since $\hat{Z}_{1}(\eta)=\eta$ does not satisfy $(6.13)$ and $\hat{Z}_{2}(\eta)=1+(1 / 2) \ln ((1+\eta) /(1-\eta))$ has infinite norm $\langle\cdot\rangle_{1}$ we get $c_{1}=0, c_{2}=0$ and hence $\hat{Z}_{h}(\eta) \equiv 0$. It follows from the symmetry conditions (6.13) that $\hat{Z}$ defined by (6.12) satisfies

$$
\int_{-1}^{1} \eta \hat{Z}(\eta) d \eta=0,
$$

which is equivalent to $(6.5)$. Hence $\hat{Z}(\theta)=Z(\cos \theta)$ coincides with the unique solution of problem (6.3). By Theorem 6.1, $Z \in H^{7 / 2}(0, \pi)$ and estimate (6.9) holds. Using Eq. (6.10) one can verify that $Z(\theta)$ satisfies the symmetry conditions (6.8). The theorem is proved.

Now we can describe our method of successive approximations. First we assume that the liquid is at rest, i.e. $v_{0}(x) \equiv 0, \varrho_{0}(x) \equiv \bar{\varrho}_{0}$, and that the domain $\Omega_{0}$ occupied by the liquid is the ball of radius $R_{0}$, i.e. $\Omega_{0}=\left\{x: r<R_{0}\right\}$. To satisfy conditions (1.5), (1.6) we have to take $\bar{\varrho}_{0}$ and $R_{0}$ such that

$$
2 \sigma / R_{0}=p\left(\bar{\varrho}_{0}\right)-p_{0}, \quad 4 \pi R_{0}^{3} \bar{\varrho}_{0} / 3=M,
$$

i.e. $R_{0}$ is the solution of the algebraic equation

$$
R_{0}\left(p\left(3 M /\left(4 \pi R_{0}^{3}\right)\right)-p_{0}\right)=2 \sigma .
$$

All the subsequent approximations $\left(v_{n}, \varrho_{n}, \Omega_{n}\right)$ will be found in symmetric form, i.e. we suppose that the functions $v_{n}, \varrho_{n}$ depend on $r, \theta$ only and satisfy the sym- 
metry conditions (5.6). The domain $\Omega_{n}$ is specified by $\Omega_{n}=\left\{x: \mathrm{r}<R_{0}+R_{n}(\theta)\right\}$, where $R_{n}$ satisfies (5.1). If $\left(v_{n}, \varrho_{n}, \Omega_{n}\right)$ are already found we take $v_{n+1}, \varrho_{n+1}$ to be a solution of the auxiliary problem (1.1) in the fixed domain $\Omega_{n}$. In addition, the function $\varrho_{n+1}$ is assumed to have the form $\varrho_{n+1}(x)=\bar{\varrho}_{n+1}+\eta_{n+1}(x)$, with $\bar{\varrho}_{n+1}=3 M /\left(2 \pi \int_{0}^{\pi} \sin \theta\left(R_{0}+R_{n}(\theta)\right)^{3} d \theta\right)$. Then at every step the condition

$$
\bar{\varrho}_{n+1}\left|\Omega_{n}\right|=M
$$

is valid. To define the domain $\Omega_{n+1}=\left\{x: r<R_{0}+R_{n+1}(\theta)\right\}$ we solve the equation

$$
\begin{aligned}
\left(\Delta^{*}+2\right) R_{n+1}= & 2 R_{0}-R_{0}^{2} \Phi^{*}\left(R_{n}\right)+R_{0}^{2} \sigma^{-1}\left(-p\left(\varrho_{n+1}\right)+p_{0}\right. \\
& \left.+\bar{n} \cdot T^{\prime}\left(v_{n+1}\right) \bar{n}\right)\left.\right|_{r=R_{0}+R_{n}(\theta)}, \quad n>1,
\end{aligned}
$$

where $\Phi^{*}(R)$ is given by (6.2).

If $\|f\|_{c^{2}\left(\Omega_{n}\right)}$ is sufficiently small (compared with $\left.\left|\Omega_{n}\right|\right)$ then problem (1.1) in $\Omega_{n}$ has a unique solution $\left(v_{n+1}, \varrho_{n+1}+\eta_{n+1}\right)$ (see Theorem 4.5) and

$$
\left\|v_{n+1}\right\|_{3, \Omega_{n}}+\left\|\eta_{n+1}\right\|_{2, \Omega_{n}} \leqq c\|f\|_{1, \Omega_{n}} .
$$

Moreover, since the initial domain $\Omega_{0}$ and $f$ satisfy the symmetry condition (5.1) and (5.4) the solution $\left(v_{n+1}, \varrho_{n+1}\right)$ also satisfies (5.6).

Consider now problem (6.18). Assume that $R_{n}(\theta)$ is a symmetric function such that $R_{n} \in H^{7 / 2}(0, \pi)$ and the conditions (6.8) are valid. Using (6.2), the representation of the normal vector $\bar{n}$ of the tensor $T^{\prime}$ in spherical coordinates and the symmetry properties of $v_{n+1}, \varrho_{n+1}$ one finds that the right-hand side of (6.18) satisfies (6.7). Moreover since $\Phi^{*}(R)$ is quadratic in $R$ (see (6.2)),

$$
\left\|\Phi^{*}\left(R_{n}\right)\right\|_{3 / 2,(0, \pi)} \leqq c\left(\left\|R_{n}\right\|_{7 / 2,(0, \pi)}\right)^{2}
$$

Further, by (6.19),

$$
\begin{aligned}
& \left\|2 R_{0}+\left.R_{0}^{2} \sigma^{-1}\left(-p\left(\varrho_{n+1}\right)+p_{0}+\bar{n} \cdot T^{\prime}\left(v_{n+1}\right) \bar{n}\right)\right|_{r=R_{0}+R_{n}(\theta)}\right\|_{3 / 2,(0, \pi)} \\
& \quad \leqq c\left(\|f\|_{1, \Omega_{n}}+g\left(\left\|R_{n}\right\|_{7 / 2,(0, \pi)}\right)\right),
\end{aligned}
$$

where $g(t)$ is small for small $t$. In deriving (6.21) we have also used (6.15) and the estimate

$$
\begin{aligned}
\left\|p\left(\bar{\varrho}_{0}\right)-p\left(\bar{\varrho}_{n+1}+\eta_{n+1}\right)\right\|_{3 / 2,(0, \pi)} & \leqq c\left(\left|\bar{\varrho}_{0}-\bar{\varrho}_{n+1}\right|+\left\|\eta_{n+1}\right\|_{2, \Omega_{n}}\right) \\
& \leqq c\left(\|f\|_{1, \Omega_{n}}+g\left(\left\|R_{n}\right\|_{7 / 2,(0, \pi)}\right)\right),
\end{aligned}
$$

which is a consequence of the formulas

$$
\bar{\varrho}_{0}=3 M / 4 \pi R_{0}^{3}, \bar{\varrho}_{n+1}=3 M /\left(2 \pi \int_{0}^{\pi} \sin \theta\left(R_{0}+R_{n}(\theta)\right)^{3} d \theta\right) .
$$

Hence by Theorem 6.2 there exists a unique solution $R_{n+1} \in H^{7 / 2}(0, \pi)$ of problem (6.18) satisfying (6.8) and the inequality

$$
\left\|R_{n+1}\right\|_{7 / 2,(0, \pi)} \leqq c\|f\|_{1, \Omega_{n}}+g_{1}\left(\left\|R_{n}\right\|_{7 / 2,(0, \pi)}\right) \text {. }
$$


Since $R_{0}(\theta) \equiv 0,(6.23)$ shows that for sufficiently small $\|f\|_{c^{2}}$ the numbers $\bar{\varrho}_{n+1}$ (mean densities) do not differ much from $\bar{\varrho}_{0}$ and the constant in (6.19) can be chosen the same for all $n$. Hence the sequences $\left\{\left\|v_{n+1}\right\|_{3, \Omega_{n}}\right\},\left\{\left\|\eta_{n+1}\right\|_{2, \Omega_{n}}\right\}$ are also bounded.

Consider the differences $\hat{v}_{n}-v_{n+1}, \hat{\eta}_{n}-\eta_{n+1}, R_{n}-R_{n+1}$ (here $\hat{v}_{n}(y)=v_{n}\left(\Phi_{n}^{-1} y\right)$, $\hat{\eta}_{n}(y)=\eta_{n}\left(\Phi_{n}^{-1} y\right), \Phi_{n}$ is the transformation which maps $\Omega_{n-1}$ onto $\left.\Omega_{n}\right)$. It follows from (6.23) and (6.19) that for sufficiently small $\|f\|_{c^{2}}$ conditions (5.8) are satisfied, and estimates (5.15), (5.16) yield

$$
\begin{gathered}
\left\|\hat{v}_{n}-v_{n+1}\right\|_{3, \Omega_{n}}+\left\|\hat{\eta}_{n}-\eta_{n+1}\right\|_{2, \Omega_{n}} \leqq c(|f|)\left\|R_{n}-R_{n-1}\right\|_{7 / 2,(0, \pi)} \\
\left\|\left.\left(-p\left(\varrho_{n}\right)+\bar{n} \cdot T^{\prime}\left(v_{n}\right) \bar{n}\right)\right|_{r=R_{0}+R_{n}(\theta)}-\left.\left(-p\left(\varrho_{n+1}\right)+\bar{n} \cdot T^{\prime}\left(v_{n+1}\right) \bar{n}\right)\right|_{r=R_{0}+R_{n}(\theta)}\right\|_{3 / 2,(0, \pi)} \\
\leqq c(|f|)\left\|R_{n}-R_{n-1}\right\|_{7 / 2,(0, \pi)} .
\end{gathered}
$$

The functions $R_{n}-R_{n+1}$ satisfy the equation

Since (see (6.2))

$$
\begin{aligned}
\left(\Delta^{*}+2\right)\left(R_{n}-R_{n+1}\right)= & -R_{0}^{2}\left(\Phi^{*}\left(R_{n-1}\right)-\Phi^{*}\left(R_{n}\right)\right)+R_{0}^{2} \sigma^{-1}\left[\left(-p\left(\varrho_{n}\right)\right.\right. \\
& \left.+\bar{n} \cdot T^{\prime}\left(v_{n}\right) \bar{n}\right)\left.\right|_{r=R_{0}+R_{n-1}(\theta)}-\left(-p\left(\varrho_{n+1}\right)\right. \\
& \left.\left.+\bar{n} \cdot T\left(v_{n+1}\right) \bar{n}\right)\left.\right|_{r=R_{0}+R_{n-1}(\theta)}\right] .
\end{aligned}
$$

$$
\begin{aligned}
\| \Phi^{*}\left(R_{n-1}\right) & -\Phi^{*}\left(R_{n}\right) \|_{3 / 2,(0, \pi)} \\
& \leqq c\left(\left\|R_{n}\right\|_{7 / 2,(0, \pi)}+\left\|R_{n-1}\right\|_{7 / 2,(0, \pi)}\right) \cdot\left\|R_{n}-R_{n-1}\right\|_{7 / 2,(0, \pi)},
\end{aligned}
$$

using (6.23) and Theorem 6.2 we get the estimate

$$
\left\|R_{n}-R_{n+1}\right\|_{7 / 2,(0, \pi)} \leqq c(|f|)\left\|R_{n-1}-R_{n}\right\|_{7 / 2,(0, \pi)} .
$$

Inequalities (6.24), (6.26) show that for $|f|$ small enough the sequences $\left\{v_{n}\right\}$, $\left\{\eta_{n}\right\}$ and $\left\{R_{n}\right\}$ have limits in the corresponding spaces. Denote these limits by $v, \eta$ and $R$ respectively.

It is evident that $v, \varrho=\bar{\varrho}+\eta$ and $\Omega=\left\{x: \mathrm{r}<R_{0}+R(\theta)\right\}$, where $\bar{\varrho}=\lim _{n \rightarrow \infty} \bar{\varrho}_{n}=3 M /\left(2 \pi \int_{0}^{\pi} \sin \theta\left(R_{0}+R(\theta)\right)^{3} d \theta\right)$, solve
Moreover, we have the estimate
\[ \|v\|_{3, \Omega}+\|\eta\|_{2, \Omega} \leqq c\|f\|_{1, \Omega} . \]

Thus, we have proved the main result of the paper.

Theorem 6.3. If the norm $\|f\|_{c^{2}}$ is small enough, if $f$ satisfies the symmetry conditions (5.4) and (1.8), if (4.6) holds and p satisfies such conditions that Eq. (6.16) is solvable. Then there exists a unique solution $(v, \varrho, \Omega)$ of problem (1.1), (1.5), (1.6). This solution satisfies the symmetry conditions (5.1), (5.6) and estimate (6.27).

Remark 6.1. To show that condition (1.8) is satisfied we show that it is satisfied on each step of the above constructed method of successive approximation. Knowing that $f_{r}=f_{r}(r, \theta), f_{\theta}=f_{\theta}(r, \theta), f_{\varphi}=0$ we have $f_{1}=\left(f_{r} \sin \theta+f_{\theta} \cos \theta\right) \cos \varphi$, $\left.f_{2}=f_{r} \sin \theta+f_{\theta} \cos \theta\right) \sin \varphi, f_{3}=f_{r} \cos \theta-f_{\theta} \sin \theta$, where $f_{i}, i=1,2,3$, are the car- 
tesian coordinates of $f$. Knowing that $\Omega_{n}$ is rotationally symmetric and $\eta_{n}=\eta_{n}(r, \theta)$ condition (1.8) implies

$$
\int_{\Omega_{n}} f_{i}(x) d x=0, \quad \int_{\Omega_{n}} \eta_{n} f_{i} d x=0, \quad i=1,2,3 .
$$

Consider

$$
\int_{0}^{\pi} \Phi(\theta) \sin \theta d \theta=\int_{0}^{\pi / 2} \Phi\left(\frac{\pi}{2}-\vartheta\right) \cos \vartheta d \vartheta+\int_{0}^{\pi / 2} \Phi\left(\frac{\pi}{2}+\vartheta\right) \cos \vartheta d \vartheta .
$$

Equations (5.4), (5.6) $)_{3}$ imply that functions $f_{r} \cos \theta, f_{\theta} \sin \theta, \eta_{n} f_{r} \cos \theta, \eta_{n} f_{\theta} \sin \theta$ satisfy the following relation

$$
\Phi\left(\frac{\pi}{2}-\vartheta\right)=-\Phi\left(\frac{\pi}{2}+\vartheta\right)
$$

which gives that (6.28) holds.

\section{A. Appendix}

In this section we prove Theorem 3.2. We use the partition of unity and notation introduced at the beginning of the proof of Lemma 4.2.

For subdomains $\widetilde{\Omega}$ such that $\overline{\widetilde{\Omega}} \cap \partial \Omega=\emptyset$ the results of [23] and local estimates for elliptic systems (see $[1,48]$ ) imply $v \in H^{3}(\widetilde{\Omega}), p \in H^{2}(\widetilde{\Omega})$ and

$$
\|v\|_{s, \tilde{\Omega}}+\|p\|_{s-1, \tilde{\Omega}} \leqq c\left(\|f\|_{s-2, \Omega}+\|g\|_{s-1, \Omega}\right), \quad s=2,3 .
$$

Hence to show (3.12) it is sufficient to estimate the solution near the boundary $S$. First we consider the case $s=2$. Then $S$ locally is expressed by function $F \in H^{5 / 2}$ which has an extension $\widetilde{F} \in H^{3}(Q)$ [see (4.18)]. Let $\widetilde{\widetilde{\Omega}} \cap S \neq \emptyset, \overline{\tilde{\omega}} \cap S \neq \emptyset$. Let us introduce new vectors $u^{\prime \prime}, \eta^{\prime \prime}$ and so on by the formulas

$$
\begin{aligned}
& u_{i}^{\prime \prime}(z)=\left.u_{i}^{\prime}(y) J^{-1}(y)\right|_{y=\Phi^{-1}(z)}, \quad i=1,2, \\
& u_{3}^{\prime \prime}(z)=\left.\left[u_{3}^{\prime}(y)-\widetilde{F}_{y_{i}}(y) u_{i}^{\prime}(y)\right] J^{-1}(y)\right|_{y=\Phi^{-1}(z)},
\end{aligned}
$$

where $J(y)=1-\tilde{F}_{y_{3}}$ is the Jacobian of the transformation $z=\Phi(y)$ (vector $u^{\prime}$ and the function $\Phi$ are defined in the proof of Lemma 4.2). Let us recall that summation convention over repeated indices be assumed. From [20] it follows that $\operatorname{div}_{z} u^{\prime \prime}(z)=\left.J^{-1}(y) \operatorname{div}_{y} u^{\prime}(y)\right|_{y=\Phi^{-1}(z)}$. For simplicity we write $w=v^{\prime \prime}, \xi=\eta^{\prime \prime}$.

Using the new variables $\{y\}$ [see (4.17)] and assuming that $\eta^{\prime}$ vanishes outside $\widetilde{\Omega}$ instead of (3.5) we obtain

$$
\frac{\mu}{2} E\left(v^{\prime}, \eta^{\prime}\right)-\left(p^{\prime}, \operatorname{div} \eta^{\prime}\right) \tilde{\Omega}=\left(f^{\prime}, \eta^{\prime}\right) \tilde{\Omega}+(\mu-v)\left(g^{\prime}, \operatorname{div} \eta^{\prime}\right) \tilde{\Omega}
$$

From (A.2) we have $v_{i}^{\prime}(y)=\left(1-\widetilde{F}_{y_{3}}(y)\right) v_{i}^{\prime \prime}(z(y)), i=1,2$,

$$
v_{3}^{\prime}(y)=\left(1-\tilde{F}_{y_{3}}(y)\right) \cdot v_{3}^{\prime \prime}(z(y))+\sum_{l=1}^{2} \tilde{F}_{y_{l}}(y) v_{l}^{\prime \prime}(z(y)) \text {. }
$$


The same formulas hold for $\eta^{\prime}$. Moreover, $\operatorname{div}_{z} v^{\prime \prime}=\left.\left(J^{-1}(y) g^{\prime}(y)\right)\right|_{y=\Phi^{-1}(z)}$. Hence, after passing to variables $z$ and integrating by parts from (A.3) one gets

$$
\frac{\mu}{2} E(w, \xi)+\frac{\mu}{2} \varepsilon(w, \xi)-(\hat{p}, \operatorname{div} \xi)_{Q}=\left(f_{1}, \xi\right)_{Q}+(\mu-v)(\operatorname{div} w, \operatorname{div} \xi)_{Q},
$$

where

$$
\begin{aligned}
& \hat{p}(z)=\left.p^{\prime}(y)\right|_{y=\Phi^{-1}(z)}, \\
& f_{1 i}(z)= {\left[f_{i}^{\prime}(y)+\widetilde{F}_{y_{i}}(y) f_{3}^{\prime}(y) J^{-1}(y)\right.} \\
&\left.+(\mu-v) \frac{\partial}{\partial z_{i}}\left(\widetilde{F}_{y_{3}} J^{-1} g^{\prime}\right)\right]\left.\right|_{y=\Phi^{-1}(z)}, \quad i=1,2, \\
& f_{13}(z)= {\left[f_{3}^{\prime}(y) J^{-1}(y)+(\mu-v) \frac{\partial}{\partial z_{3}}\left(\widetilde{F}_{y_{3}} J^{-1} g^{\prime}\right)\right], } \\
& \varepsilon(w, \xi)= \int_{Q}\left[p_{k l}^{i j} w_{i z_{k}} \xi_{j z_{l}}+p_{k}^{i j} w_{i z_{k}} \xi_{j}+\bar{p}_{k}^{i j} w_{i} \xi_{j z_{k}}+p^{i j} w_{i} \xi_{j}\right] J^{-1} d z, \\
& p_{k l}^{i j}=p_{4}^{(1)}(\nabla \widetilde{F}), \quad p_{k}^{i j}, \bar{p}_{k}^{i j}=p_{3}^{(2)}(\nabla \widetilde{F}) D^{2} \widetilde{F}+p_{2}^{(3)}(\nabla \widetilde{F}), \\
& p^{i j}=p_{2}^{(4)}(\nabla \widetilde{F}) D^{2} \widetilde{F} D^{2} \widetilde{F},
\end{aligned}
$$

$p_{l}^{(m)}$ are polynomials of degree $l$ with no constant terms.

Put in (A.4) $\zeta \xi$ in place of $\xi$, where $\zeta$ is a smooth function such that $\zeta=1 \quad$ if $\quad\left|z_{i}\right| \leqq d / 4, \quad i=1,2, \quad z_{3} \leqq d / 4, \quad$ and $\quad \zeta=0 \quad$ if $\quad\left|z_{i}\right| \geqq d / 2, \quad z_{3} \geqq d / 2$. Let $\tilde{w}=\zeta w, \tilde{p}=\hat{p} \zeta, \widetilde{f}_{1}=f_{1} \zeta$. Then instead of (A.4) one gets

$$
\frac{\mu}{2} E(\tilde{w}, \xi)+\frac{\mu}{2} \varepsilon(\tilde{w}, \xi)-(\tilde{p}, \operatorname{div} \xi)_{Q}=\Phi(\xi)+(\mu-v)(\operatorname{div} \tilde{w}, \operatorname{div} \xi)_{Q},
$$

where

$$
\begin{gathered}
\Phi(\xi)=-\frac{\mu}{2} E^{\prime}(w, \zeta, \xi)-\frac{\mu}{2} \varepsilon^{\prime}(w, \zeta, \xi)+(\hat{p}, \nabla \zeta \cdot \xi)_{Q}+\left(\widetilde{f_{1}}, \xi\right)_{Q} \\
+(\mu-v)(\nabla \zeta \operatorname{div} w, \xi)_{Q}+(\mu-v)(\nabla(w \cdot \nabla \zeta), \xi)_{Q}, \\
E^{\prime}(w, \zeta, \xi)=\int_{Q}\left[T_{i j}(w)\left(\zeta_{z_{j}} \xi_{i}+\zeta_{z_{i}} \xi_{j}\right)-\left(\zeta_{z_{i}} w_{j}+\zeta_{z_{j}} w_{i}\right) T_{i j}(\xi)\right] d z \\
\varepsilon^{\prime}(w, \zeta, \xi)=\int_{Q}\left[p_{k l}^{i j}\left(w_{i z_{k}} \zeta_{z_{l}} \xi_{j}-w_{i} \zeta_{z_{k}} \xi_{j_{z}}\right)-\left(p_{k}^{i j}+\bar{p}_{k}^{i j}\right) w_{i} \zeta_{z_{k}} \xi_{j}\right] J^{-1} d z
\end{gathered}
$$

To prove Theorem 3.2 for $s=0$ we regularize the boundary, i.e. we assume that $\widetilde{F}_{m} \in C^{\infty}$ and $\left\|\widetilde{F}_{m}-\widetilde{F}\right\|_{3} \rightarrow 0, m \rightarrow \infty$. Consider the integral identity

$$
\frac{\mu}{2} E\left(w_{m}, \xi\right)+\frac{\mu}{2} \varepsilon_{m}\left(w_{m}, \xi\right)-\left(q_{m}, \operatorname{div} \xi\right)_{Q}=\Phi(\xi)+(\mu-v)(\operatorname{div} \tilde{w}, \operatorname{div} \xi)_{Q}
$$


where $q_{m}=\tilde{p}_{m}$ and

$$
\begin{gathered}
\varepsilon_{m}(w, \xi)=\int_{Q}\left(p_{k m}^{i j}(m) w_{i z_{k}} \xi_{j z_{l}}+\left(p_{k}^{i j}(m) w_{i z_{k}} \xi_{j}+\bar{p}_{k}^{i j}(m) w_{i} \xi_{j z_{k}}+p^{i j}(m) w_{m i} \xi_{j}\right) J^{-1} d z\right. \\
p_{k l}^{i j}=p_{4}^{(1)}\left(\nabla \widetilde{F}_{m}\right) \text { and so on } .
\end{gathered}
$$

From the definition of $\Phi$ we have

$$
|\Phi(\xi)| \leqq c\left(\|\hat{p}\|_{0}+\|w\|_{1}+\left\|\tilde{f}_{1}\right\|_{0}\right)\|\xi\|_{1} \leqq c\left(\|f\|_{0}+\|g\|_{0}\right)\|\xi\|_{1}
$$

To obtain (A.10) we have used (3.11) and estimates

$$
\begin{gathered}
\left|E^{\prime}(w, \zeta, \xi)\right| \leqq c\|w\|_{1}\|\xi\|_{1}, \\
\left|\varepsilon^{\prime}(w, \zeta, \xi)\right| \leqq c\left(\|\tilde{F}\|_{3}\right)\|w\|_{1}\|\xi\|_{1},
\end{gathered}
$$

which follow from (A.8), defintion of the coefficients $p_{k l}^{i j}, p_{k}^{i j}, \bar{p}_{k}^{i j}, p^{i j}$ and imbedding theorems. Moreover,

$$
\left|\varepsilon_{m}\left(w_{m}, \xi\right)\right| \leqq c(d)\left\|w_{m}\right\|_{1}\|\xi\|_{1},
$$

where $c(d) \rightarrow 0$ with $d \rightarrow 0$. To prove (A.12) we use $\left\|\tilde{F}_{m}-\tilde{F}\right\|_{3} \rightarrow 0$ and the fact that $\nabla \tilde{F}$ is small in a neighbourhood of the point $z=0$. Since the term $\varepsilon_{m}\left(w_{m}, \xi\right)$ defines a small operator we can use standard arguments (see $[47,53,54]$ ) to prove the existence of a unique solution $\left(w_{n}, q_{m}\right)$ satisfying (A.9) and such that

$$
\begin{gathered}
w_{m} \in H^{1}(Q), \quad q_{m} \in \hat{L}_{2}(Q), \quad \operatorname{div} w_{m}=q_{1} \equiv \operatorname{div} \tilde{w}, \\
\left.w_{m}\right|_{\partial Q \backslash\left(z: z_{3}=0\right)}=0,\left.\quad w_{m 3}\right|_{z_{3}=0}=0, \\
\left\|w_{m}\right\|_{1}+\left\|q_{m}\right\|_{0} \leqq c\left(\|f\|_{0}+\|g\|_{0}+\left\|g_{1}\right\|_{0}\right) \leqq c\left(\|f\|_{0}+\|g\|_{0}\right) .
\end{gathered}
$$

Let $\xi$ have a compact support in $Q$. Integrating by parts we can reduce the right-hand side of $\Phi(\xi)$ from (A.9) to the form $\Phi(\xi)=(\tilde{\Phi}, \xi)_{Q}$, where $\tilde{\Phi} \in L_{2}(Q)$. Moreover, since the coefficients of $\varepsilon_{m}$ are smooth and $g_{1}=\operatorname{div} \tilde{w}=\zeta g+\nabla \zeta \cdot w \in H^{1}(Q)$ we can prove (in the same way as in [23], the additional term $\varepsilon_{m}$ doesn't imply any difference) that $w_{m} \in H^{2}(\widetilde{Q}), q_{m} \in H^{1}(\widetilde{Q})$ for every $\widetilde{Q}$ such that $\tilde{Q} \subset Q$. Integrating by parts in (A.9) with $\xi$ such that supp $\xi \subset Q$ we get that $\left(w_{m}, q_{m}\right)$ satisfies almost everywhere in $Q$ the following system of equations

$$
\begin{gathered}
-\mu \Delta_{z} w_{m}-v \nabla_{z} \operatorname{div}_{z} w_{m}+\sum_{|\alpha| \leqq 2} \sum_{k=1}^{3} b_{k}^{(\alpha)} D_{z}^{\alpha} w_{m k}+\nabla_{z} q_{m}=\tilde{\Phi} \\
\operatorname{div}_{z} w_{m}=g_{1}
\end{gathered}
$$

where the sum in (A.15) corresponds to the term $\varepsilon_{m}$ and the coefficients $b_{k}^{(\alpha)},|\alpha|=2$, are small as $d \rightarrow 0$. To estimate $w_{m}, q_{m}$ near the boundary $z_{3}=0$ one can use the arguments from [47]. First, from the integral identity (A.9) one gets $w_{m z \tau} \in L_{2}\left(Q^{\prime}\right)$, $q_{m \tau} \in L_{2}\left(Q^{\prime}\right)$, where $Q^{\prime}=\operatorname{supp} \zeta$ and the index $\tau$ means the derivatives with respect to $z_{i}, i=1,2$. Then expressing $w_{m z_{3} z_{3}}, q_{m z_{3}}$ from the "Stokes" equations (A.15) one gets $w_{m z_{3} z_{3}}, q_{m z_{3}} \in L_{2}(Q)$. Hence $w_{m} \in H^{2}\left(Q^{\prime}\right), q_{m} \in H^{1}\left(Q^{\prime}\right)$. Note once more, that in these considerations the smoothness of function $\widetilde{F}_{m}$ has been used. 
Now we estimate $\left\|w_{m}\right\|_{2, Q^{\prime}}\left\|q_{m}\right\|_{1, Q}$, using only that $\widetilde{F}_{m} \in H^{3}$. To do this we put $\xi=\zeta\left(\delta_{h}^{l} \tilde{w}_{m}\right)_{\tau}, l=1,2, \tilde{w}_{m}=\zeta w_{m}, q_{m}=\zeta q_{m}$ into (A.9). Then we get

$$
\begin{aligned}
& \frac{\mu}{2} E\left(\tilde{w}_{m},\left(\delta_{h}^{l} \tilde{w}_{m}\right)_{\tau}\right)+ \frac{\mu}{2} \varepsilon_{m}\left(\tilde{w}_{m},\left(\delta_{h}^{l} \tilde{w}_{m}\right)_{\tau}\right)-\left(\tilde{q}_{m}, \operatorname{div}\left(\delta_{h}^{l} \tilde{w}_{m}\right)_{\tau}\right)_{Q} \\
&= \Phi\left(\zeta\left(\delta_{h}^{l} \tilde{w}_{m}\right)_{\tau}\right)+\left(q_{m} \nabla \zeta,\left(\delta_{h}^{l} \tilde{w}_{m}\right)_{\tau}\right)_{Q} \\
&+(\mu-v)\left(g_{1}, \operatorname{div}\left(\zeta\left(\delta_{h}^{l} \tilde{w}_{m}\right)_{\tau}\right)_{Q}\right. \\
&-\frac{\mu}{2} E^{\prime}\left(w_{m}, \zeta,\left(\delta_{h}^{l} \tilde{w}_{m}\right)_{\tau}\right)-\frac{\mu}{2} \varepsilon^{\prime}\left(w_{m}, \zeta,\left(\delta_{h}^{l} \tilde{w}_{m}\right)_{\tau}\right) \\
& \equiv A\left(\left(\delta_{h}^{l} \tilde{w}_{m}\right)_{\tau}\right), \\
& \frac{\mu}{2} E\left(\tilde{w}_{m},\left(\delta_{h}^{l} \tilde{w}_{m}\right)_{\tau}\right)=-\frac{\mu}{2} E\left(\tilde{w}_{m \tau}, \delta_{h}^{l} \tilde{w}_{m}\right) .
\end{aligned}
$$

Estimating the right-hand side of (A.16) we get

$$
\begin{aligned}
|A(\xi)| & \leqq c\left(\left\|w_{m}\right\|_{1}+\left\|g_{1 \tau}\right\|_{0}+\left\|q_{m}\right\|_{0}+\|f\|_{0}+\|g\|_{0}\right)\left\|\delta_{h}^{l} \nabla_{z} \tilde{w}_{m}\right\|_{0} \\
& \leqq c\left(\|f\|_{0}+\|g\|_{1}\right)\left\|\delta_{h}^{l} \nabla_{z} \tilde{w}_{m}\right\|_{0} .
\end{aligned}
$$

To prove (A.17) we integrate by parts with respect to $\tau$ in terms $E^{\prime}, \varepsilon^{\prime}$ and use estimates (A.10), (A.11), (A.14), and (3.11).

Now we estimate $\varepsilon_{m}\left(\tilde{w}_{m}, \xi\right)$. Using the form of $\varepsilon_{m}$ we have

$$
\begin{aligned}
\left|\varepsilon_{n}\left(\tilde{w}_{m},\left(\delta_{h}^{l} \tilde{w}_{m}\right)_{\tau}\right)\right|= & \left|\int_{Q}\left(p_{k l}^{i j}(m) \tilde{w}_{m i z_{k}} J^{-1}\right)_{\tau} \delta_{h}^{l} \tilde{w}_{m j z} d z\right| \\
& +\left|\int_{Q} p_{k}^{i j}(m) \tilde{w}_{m i z_{k}} J^{-1} \delta_{h}^{l} \tilde{w}_{m j \tau} d z\right| \\
& +\left|\int_{Q}\left(\bar{p}_{k}^{i j}(m) \tilde{w}_{m i} J^{-1}\right)_{\tau} \delta_{h}^{l} \tilde{w}_{m j z_{k}} d z\right| \\
& +\left|\int_{Q} p^{i j}(m) \tilde{w}_{m i} J^{-1} \delta_{h}^{l} \tilde{w}_{m j \tau} d z\right| \\
\leqq & c\left\{\left|\left(p_{k l}^{i j}(m)\right)_{\tau}\right|_{4}\left|J^{-1}\right|_{\infty}\left|\tilde{w}_{m z}\right|_{4}+\left|p_{k l}^{i j}(m)\right|_{\infty}\left|\left(J^{-1}\right)_{\tau}\right|_{4}\left|\tilde{w}_{m z}\right|_{4}\right. \\
& +\left|p_{k l}^{i j}(m)\right|_{\infty}\left|J^{-1}\right|_{\infty}\left|\tilde{w}_{m z \tau}\right|_{2} \\
& +\left|p_{k}^{i j}(m)\right|_{4}\left|J^{-1}\right|_{\infty}\left|\tilde{w}_{m z}\right|_{4}+\left|\left(\bar{p}_{k}^{i j}(m)\right)_{\tau}\right|_{2}\left|J^{-1}\right|_{\infty}\left|\tilde{w}_{m}\right|_{\infty} \\
& +\left|\bar{p}_{k}^{i j}(m)\right|_{4}\left|J^{-1}\right|_{\infty}\left|\tilde{w}_{m z}\right|_{4}+\left|\bar{p}_{k}^{i j}(m)\right|_{4}\left|\left(J^{-1}\right)_{\tau}\right|_{4}\left|\tilde{w}_{m}\right|_{\infty} \\
& \left.+\left|J^{-1}\right|_{\infty}\left|p^{i j}(m)\right|_{2}\left|\tilde{w}_{m}\right|_{\infty}\right\}\left\|\tilde{w}_{m z \tau}|| \sum_{0} \leqq c(d)\right\| \tilde{w}_{m}\left\|_{2}\right\| \tilde{w}_{m z \tau} \|_{0},
\end{aligned}
$$

where $c(d) \rightarrow 0$ with $d \rightarrow 0$. Above, we have used that $\tilde{w}_{m} \in H^{2}$ and imbedding theorems. To prove estimates $\left|p_{k l}^{i j}\right|_{\infty^{\prime}}\left|\left(p_{k l}^{i j}\right)_{\tau}\right|_{4} \leqq c(d)$ and so on the fact that, $\nabla \widetilde{F}$ is small in neighbourhood of $z=0$ and the estimate $|u|_{4, Q} \leqq c(d)|u|_{6, Q} \leqq c(d)\|u\|_{1}$ which follows from the imbedding $H^{1} \subset L_{6}$ have been used. 
Consider the term $\left(\tilde{q}_{m}, \operatorname{div} \delta_{h}^{l} \tilde{w}_{m \tau}\right)_{Q}$. Since $\operatorname{div} \tilde{w}_{m}=\nabla \zeta \cdot w_{m}+\zeta g_{1}$ we get

$$
\begin{aligned}
\left|\left(\tilde{q}_{m}, \operatorname{div} \delta_{h}^{l} \tilde{w}_{m \tau}\right)_{Q}\right| & =\left|-\left(\tilde{q}_{m \tau}, \delta_{h}^{l}\left(\nabla \zeta \cdot w_{m}\right)\right)_{Q}-\left(\tilde{q}_{m \tau}, \delta_{h}^{l}\left(\zeta g_{1}\right)\right)_{Q}\right| \\
& \leqq \varepsilon\left\|\tilde{q}_{m \tau}\right\|_{0}+c(\varepsilon)\left(\left\|w_{m}\right\|_{1}+\left\|g_{1 \tau}\right\|_{0}\right) .
\end{aligned}
$$

Passing to a limit as $h \rightarrow 0$ from (A.16)-(A.19) one derives the estimate

$$
\left\|\tilde{w}_{m z \tau}\right\|_{0} \leqq c\left(\|f\|_{0}+\|g\|_{1}\right)+\varepsilon\left\|\tilde{q}_{m \tau}\right\|_{0}+c(d)\left\|\tilde{w}_{m}\right\|_{0} .
$$

In the same way as in [47] we can get the estimate for $\left\|\tilde{q}_{m \tau}\right\|_{0}$, i.e. we put $\xi=\zeta \delta_{h}^{l} \Phi_{\tau}$ into (A.9), where $\Phi$ is a solution of the problem

$$
\operatorname{div} \Phi=\tilde{q}_{m},\left.\quad \Phi\right|_{z_{3}}=0
$$

in the half-space $z_{3}=0$. Repeating the above estimates one gets

$$
\begin{aligned}
\left\|\tilde{q}_{m \tau}\right\|_{0} & \leqq c\left(\left\|\tilde{q}_{m}\right\|_{0}+\left\|\tilde{w}_{m}\right\|_{1}+\|f\|_{0}+\|g\|_{1}\right)+c\left\|\tilde{w}_{m z \tau}\right\|_{0}+c(d)\left\|\tilde{w}_{m}\right\|_{2} \\
& \leqq c\left(\|f\|_{0}+\|g\|_{1}\right)+c\left\|\tilde{w}_{m z \tau}\right\|_{0}+c(d)\left\|\tilde{w}_{m}\right\|_{0} .
\end{aligned}
$$

Now we can estimate the derivatives $\tilde{w}_{m z_{3} z_{3}}, \tilde{q}_{m z_{3}}$ calculating them from Eqs. (A.15) (see the end of Sect. 4 in [47]). Hence one gets

$$
\left\|\tilde{w}_{m z_{3} z_{3}}\right\|_{0}+\left\|\tilde{q}_{m z_{3}}\right\|_{0} \leqq c\left(\|f\|_{0}+\|g\|_{1}+\left\|\tilde{w}_{m z \tau}\right\|_{0}+\left\|\tilde{q}_{m \tau}\right\|_{0}\right) \text {. }
$$

From (A.20)-(A.22) we obtain (if $\varepsilon$ and $d$ are small)

$$
\left\|\tilde{w}_{m}\right\|_{2}+\left\|\tilde{q}_{m}\right\|_{1} \leqq c\left(\|f\|_{0}+\|g\|_{1}\right) .
$$

Therefore from (A.14) there exists a subsequence $\left\{w_{m}, q_{m}\right\}$ (for simplicity we denote it in the same way) weakly converging in $H^{1}(Q) \times L_{2}(Q)$. From (A.23) it converges weakly in $H^{2}\left(Q^{\prime}\right) \times H^{1}\left(Q^{\prime}\right)$ and strongly in $H^{1}\left(Q^{\prime}\right) \times L_{2}\left(Q^{\prime}\right)$. Let $(U, P) \in\left(H^{1}(Q) \times L_{2}(Q)\right) \cap\left(H^{2}\left(Q^{\prime}\right) \times H^{1}\left(Q^{\prime}\right)\right)$ be a limit of this subsequence. We will prove that $(U, P)$ satisfies the identity (A.6). Let $m \rightarrow \infty$ in (A.9). It is sufficient to consider the term $\varepsilon_{m}\left(w_{m}, \xi\right)$ only:

$$
\begin{aligned}
\left|\varepsilon_{m}\left(\tilde{w}_{m}, \xi\right)-\varepsilon(U, \xi)\right|= & \mid{ }_{Q}\left\{\left(p_{k l}^{i j}(m)-p_{k l}^{i j}\right) \tilde{w}_{m i z_{k}} \xi_{j z_{l}}\right. \\
& +p_{k l}^{i j}\left(\tilde{w}_{m i z_{k}}-\tilde{U}_{i z_{k}}\right) \xi_{j z_{l}}+\left(p_{k}^{i j}(m)-p_{k}^{i j}\right) \tilde{w}_{m i z_{k}} \xi_{j} \\
& +p_{k}^{i j}\left(\tilde{w}_{m i z_{k}}-\tilde{U}_{i z_{k}}\right) \xi_{j} \\
& +\left(\bar{p}_{k}^{i j}(m)-\bar{p}_{k}^{i j}\right) \tilde{w}_{m i} \xi_{j z_{k}}+\bar{p}_{k}^{i j}\left(\tilde{w}_{m i}-\tilde{U}_{i}\right) \xi_{j z_{k}} \\
& \left.+\left(p^{i j}(m)-p^{i j}\right) \tilde{w}_{m i} \xi_{j}+p^{i j}\left(\tilde{w}_{m i}-\tilde{U}_{i}\right) \xi_{j}\right\} J^{-1} d z \mid \\
\leqq & c\left|p_{k l}^{i j}(m)-p_{k l}^{i j}\right|_{\infty}\left|\tilde{w}_{m z}\right|_{2}\|\xi\|_{0}+c\left|p_{k}^{i j}(m)-p_{k}^{i j}\right|_{4}\left\|\tilde{w}_{m z}\right\|_{0}|\xi|_{4} \\
& +c\left|\bar{p}_{k}^{i j}(m)-\bar{p}_{k}^{i j}\right|_{4}\left|\tilde{w}_{m}\right|_{4}\left\|\xi_{z}\right\|_{0}+c\left|p^{i j}(m)-p^{i j}\right|_{2}\left|\tilde{w}_{m}\right|_{4}|\xi|_{4} \\
& \left.+\left|\bar{p}_{k}^{i j}\right|_{4}\left|w_{m}-U\right|_{4}\left|\xi_{z}\right|_{2}+\left|p^{i j}\right|_{3}\left|w_{m}-U\right|_{2}|\xi|_{6}\right) \\
& +\left|\int_{Q}\left\{\left(p_{k l}^{i j}\left(\tilde{w}_{m i z_{k}}-\tilde{U}_{i z_{k}}\right) \xi_{j z_{k}}+p_{k}^{i j}\left(\tilde{w}_{m i z_{k}}-\tilde{U}_{i z_{k}}\right) \xi_{j}\right) J^{-1}\right\} d z\right| .
\end{aligned}
$$


The terms in the brackets tend to zero because $\left\|\widetilde{F}_{m}-\tilde{F}\right\|_{3} \rightarrow 0$ and the imbedding $H^{1} \subset L_{q}, q<6$, is compact. The last integral in the right of (A.24) is a linear functional on $H^{1}(Q)$, so it tends to zero. Finally after passing to the limit in (A.9) we obtain

$$
\frac{\mu}{2} E(U, \xi)+\frac{\mu}{2} \varepsilon(U, \xi)-(P, \operatorname{div} \xi)_{Q}=\Phi(\xi)+(\mu-v)(\operatorname{div} \tilde{w}, \operatorname{div} \xi)_{Q}
$$

Subtracting (A.25) from (A.6) and taking in the resulting identity $\xi=\tilde{w}-U$ one gets $($ since $\operatorname{div}(\tilde{w}-U)=0)$

$$
\frac{\mu}{2} E(\tilde{w}-U, \tilde{w}-U)+\frac{\mu}{2} \varepsilon(\tilde{w}-U, \tilde{w}-U)=0 .
$$

Estimating the term $\varepsilon(\tilde{w}-U, \tilde{w}-U)$ by (A.12) we obtain

$$
E(\tilde{w}-U, \tilde{w}-U) \leqq c(d)\|\tilde{w}-U\|_{1} .
$$

Hence for sufficiently small $d$ we have $\tilde{w}-U \equiv 0$, i.e. $\tilde{w}=U \in H^{2}\left(Q^{\prime}\right)$. From the Stokes system we get $\tilde{q} \in H^{1}\left(Q^{\prime}\right)$. The estimate (3.12), $s=2$, follows from (A.23). Therefore Theorem 3.2 for $s=2$ is proved.

Now we shall obtain an estimate for $v \in H^{3}(\Omega)$ and $p \in H^{2}(\Omega)$. In this case $S \in H^{7 / 2}$. Applying transformations (4.17) and (4.18) problem (3.1), (3.2) can be written in the form

$$
\begin{gathered}
A_{z} \tilde{v}+\nabla_{z} \tilde{p}=\left(A_{z}-\hat{A}\right) \tilde{v}+\left(\nabla_{z}-\hat{\nabla}\right) \tilde{p}+\hat{A} \tilde{v}-\hat{A} \hat{v} \hat{\zeta} \quad \text { in } Q, \\
+\hat{\nabla} \tilde{p}-\hat{\nabla} \hat{p} \hat{\zeta}+\tilde{f}, \quad \operatorname{div}_{z} \tilde{v}=\operatorname{div}_{z} \tilde{v}-\hat{\operatorname{div}} \hat{v} \hat{\zeta}+\tilde{g} \text { in } Q, \\
\hat{v} \cdot \bar{n}_{z}=\hat{v} \bar{n}_{z}-\hat{v} \hat{\bar{n}} \quad \text { on } \hat{S}, \\
\bar{\tau}_{z} T_{z}^{\prime}(\tilde{v}) \bar{n}_{z}=\bar{\tau}_{z} T_{z}^{\prime}(\tilde{v}) \bar{n}_{z}-\hat{\bar{\tau}} \hat{T}^{\prime}(\hat{v}) \hat{\bar{n}} \bar{\zeta} \quad \text { on } \hat{S}, \\
\tilde{v} \bar{n}_{z}=0 \quad \text { on } \partial Q \backslash \hat{S}, \\
\bar{\tau}_{z} T_{z}^{\prime}(\tilde{v}) \bar{n}_{z}=0 \text { on } \partial Q \backslash \hat{S},
\end{gathered}
$$

where the same notations as in the proof of Lemma 4.2 [see also problem (4.40)] are used. Applying the operator $\delta_{l}^{k}(k=1,2)$ to (A.21), using the fact that $z=z(y) \in H^{4}(\widetilde{\Omega}), v \in H^{2}, p \in H^{1}$ and the estimates for the Stokes problem in the half space (see [1, 48]; it was shown in [48] that the Stokes system is an elliptic DouglisNirenberg system and the boundary conditions (3.2) satisfy the compatibility condition) one has

$$
\begin{aligned}
\left\|\delta_{h}^{k} \tilde{v}\right\|_{2, Q}+\left\|\delta_{h}^{k} \tilde{p}\right\|_{1, Q} \leqq & c d\left(\left\|\delta_{h}^{k} \tilde{v}\right\|_{2, Q}+\left\|\delta_{h}^{k} \tilde{p}\right\|_{1, Q}\right) \\
& +\left[P\left(\left\|z_{y}\right\|_{3, Q}\right)+c(\zeta)\right]\left(\|v\|_{2}+\|p\|_{1}\right) \\
& +c\left(\|f\|_{1}+\|g\|_{2}\right)
\end{aligned}
$$

Therefore for sufficiently small $d$ one obtains in the limit as $h \rightarrow 0$

$$
\left\|\tilde{v}_{\tau}\right\|_{2, Q}+\left\|\tilde{p}_{\tau}\right\|_{1, Q} \leqq c\left(\|v\|_{2}+\|p\|_{1}+\|f\|_{1}+\|g\|_{2}\right) \text {. }
$$

The derivatives $\tilde{v}_{z_{3} z_{3} z_{3}}, \tilde{p}_{z_{3} z_{3}}$ are estimated in the same way as in the proof of Theorem 2 in [47]. This concludes the proof of Theorem 3.2. 
Acknowledgements. The authors are greatly indebted to Prof. V.A. Solonnikov for showing them the proof of Theorem 3.2.

\section{References}

1. Agmon, S., Douglis, A., Nirenberg, L.: Estimates near the boundary for solutions of elliptic partial differential equations satisfying general boundary conditions. II. Comm. Pure Appl. Math. 17, 35-72 (1964)

2. Allain, G.: Small time existence for the Navier-Stokes equations with a free surface. Ecole Polytechnique. Rapport interue. 135, 1-24 (1985)

3. Antonovski, L.K.: Complex representation of solutions to Navier-Stokes equations. Dokl. Akad. Nauk. SSSR 261, 829-832 (1981) (in Russian)

4. Antonovski, L.K.: Exact solutions of a free boundary problem for the Stokes system. Dokl. Akad. SSSR 270, 1082-1084 (1983) (in Russian)

5. Beale, J.T.: The initial value problem for the Navier-Stokes equations with a free boundary. Comm. Pure Appl. Math. 31, 359-392 (1980)

6. Beale, J.T.: Large-time regularity of viscous surface waves. Arch. Rat. Mech. Anal. 84, 307-352 (1984)

7. Beirao da Veiga, H.: Stationary motion and incompressible limit for compressible viscous fluid. Preprint of University of Wisconsin, Mathematical research center, MRC 2883 (1985)

8. Beirao da Veiga, H.: On a stationary transport equation. Ann. Univ. Ferrara, Sez. VII, 32, 79-91 (1986)

9. Beirao da Veiga, H.: An $L^{p}$-theory for the $n$-dimensional, stationary, compressible NavierStokes equations, and the incompressible limit for compressible fluids. The equilibrium solutions. Commun. Math. Phys. 109, 229-248 (1987)

10. Bemelmans, J.: Gleichgewichtsfiguren zäher Flüssigkeiten mit Oberflächenspannung. Analysis 1, 241-282 (1981)

11. Bemelmans, J.: A note on the interpretation of closed $H$-surfaces in physical terms. Manuscripta Math. 36, 347-354 (1981)

12. Bemelmans, J.: On a free boundary problem for the stationary Navier-Stokes equations. Ann. Inst. Henri Poincaré 4(6), 517-547 (1987)

13. Bemelmans, J.: Liquid drops in a viscous fluid under the influence of gravity and surface tension. Manuscripta Math. 36, 105-123 (1981)

14. Bemelmans, J.: Nonstationary viscous fluid flow generated by surface tension. I. Existence of classical solutions. Preprint no 509, Bonn 1982

15. Erunova, I.B.: Investigation of the problem of the stationary motion of two liquids in a container. Dokl. Akad. Nauk SSSR 279, 53-58 (1984) (in Russian)

16. Erunova, I.B.: Solvability of a free boundary problem for two fluids in a container. Vestnik Leningrad. Univ. 2, 9-16 (1986) (in Russian)

17. Friedrichs, K.O.: Symmetric positive linear differential equations, Comm. Pure Appl. Math 11, 333-418 (1958)

18. Fuita-Yashima, H.: Probleme de la surface libre de l'equation de Navier-Stokes-cas stationnaire et cas periodique, preprint

19. Gilbarg, D., Trudinger, N.S.: Elliptic partial differential equations of second order. Berlin Heidelberg New York: Springer 1977

20. Kapitanskii, L.V., Pileckas, K.: Certain problems of vector analysis. Zap. Nauchn. Sem. LOMI 138, 65-85 (1984) (in Russian) [transl. J of Soviet Math. 32, 197-203 (1984)]

21. Ladyzhenskaya, O.A., Solonnikov, V.A.: On some problems of vector analysis and on generalized formulations of boundary value problems for the Navier-Stokes equations. Zap. Nauchn. Sem. LOMI 59, 81-116 (1976) (in Russian)

22. Ladyzhenskaya, O.A., Osmolowski, V.G.: On the free surface of a fluid layer over a solid sphere. Vestnik Leningrad. Univ. Mat. Mekh. Astronom. 13, 25-30 (1976) (in Russian) 
23. Ladyzhenskaya, O.A.: The mathematical theory of viscous incompressible flow. New York: Gordon and Breach 1969

24. Landau, L., Lifschitz, E.: Mechanics of continuum media. Moscow 1954 (in Russian) (English transl. Pergamon Press: Oxford 1959)

25. Maz'ya, V.G., Shaposhnikova, T.O.: Multipliers in spaces of differentiable functions. Leningrad 1986 (in Russian)

26. Maz'ya, V.G., Plamenevskii, B.A., Stupelis, L.I.: A three-dimensional free boundary problem. Diff. Eq. and their Appl., Inst. of Math. and Cybern. Acad. Sci. Lit. SSR, Vilnius 1979, 9-153 (in Russian)

27. Nishida, T.: Equations of fluid dynamics-free surface problems, Comm. Pure Appl. Math. 39, 221-238 (1986)

28. Osmolovskii, V.G.: The free surface of a drop in a symmetric force field. Zap. Nauchn. Sem. LOMI 52, 160-174 (1975) (in Russian)

29. Pileckas, K. : Solvability of a problem of plane motion of a viscous incompressible fluid with noncompact free boundary. Diff. Equ. Appl. Inst. of Math. Cybern. Acad. Sci. Lit. SSR 30, 57-96, 1981 (in Russian)

30. Pileckas, K.: On the problem of motion of heavy viscous incompressible fluid with noncompact free boundary. Lith. Math. J. 28, 2, 315-333, 1988 (in Russian). Zap. Nauchn. Sem. LOMI 110, 174-179, 1981 (in Russian)

31. Pileckas, K.: On plane motion of a viscous incompressible capillary liquid with a noncompact free boundary. Trans. Fluid Mech. (1988) (to be published)

32. Pileckas, K., Specovius-Neugebauer, M.: Solvability of a non-compact free boundary problem for the stationary Navier-Stokes system. I. Lith. Math. J. 29, 3 (1989); II. Lith. Math. J. 29, 4 (1989) (to be published) (in Russian)

33. Pileckas, K., Solonnikov, V.A.: On stationary Stokes and Navier-Stokes systems in a open infinite channel. I. Lith. Math. J. 29, 1 (1989); II. Lith. Math. J. 29, 2 (1989) (in Russian)

34. Pukhnachov, V.V.: Plane stationary free boundary problem for Navier-Stokes equation. Zh. Prikl. Mekh. i Tekhn. Fiz. 3, 91-102 (1972) (in Russian)

35. Pukhnachov, V.V.: Mises' variables in free boundary problems for the Navier-Stokes equations. Dokl. Akad. Nauk SSSR 210, 298-301 (1973) (in Russian)

36. Pukhnachov, V.V.: Free boundary problems for Navier-Stokes equations, Doctoral dissertation, Novosibirsk 1974 (in Russian)

37. Pukhnachov, V.V.: Regularity of stationary solutions of Navier-Stokes equations near a free boundary. Dinamika sploshn. Sredy. 15, 133-144 (1973)

38. Rivkind, V.Ya.: Theoretical justification of a method of successive approximations for stationary problems of mechanics a viscous fluid with free surfaces of separation. Zap. Nauchn. Sem. LOMI 115, 228-235 (1982) (in Russian)

39. Rivkind, V.Ya.: A priori estimates and the method of successive approximations for the motion of a drop. Trudy Mat. Inst. Steklov 159, 150-166, 1983 (in Russian)

40. Rivkind, V.Ya., Fridman, N.B.: On the Navier-Stokes equations with discontinuous coefficients. Zap. Nauchn. Sem. LOMI 38, 137-148 (1973) (in Russian)

41. Sattinger, D.: On the free surface of a viscous fluid. Proc. R. Soc. London A 349, 183-204 (1976)

42. Shubin, M.A.: Pseudodifferential operators and spectral theory, Moscow 1978 (in Russian)

43. Socolescu, D.: Existenz und Eindeutigkeitsbeweis für ein freies Randwertproblem für die stationären Navier-Stokesschen Bewegungsgleichungen. ARMA 73, 3, 191-243 (1980)

44. Sokolescu, D.: On one free boundary problem for the stationary Navier-Stokes equations. Methoden und Verfahren der mathematischen Physik 18, Free and mixed boundary value problems, pp 117-140, 1979

45. Sokolowsky, J.: Solvability of a two-dimensional problem for the motion of two viscous incompressible liquids with non-compact free boundaries. Zap. Nauchn. Sem. LOMI 163, 146-153 (1987) (in Russian)

46. Sokolowsky, J., Berman, J.: Leitlinientechnik zur Berechnung von Strömungen nichtlinearviskoser Medien bei Beschichtungsvorgängen. Technische Mechanik 7, 21-29 (1986) 
47. Solonnikov, V.A., Shchadilov, V.E.: On a boundary value problem for a stationary system of Navier-Stokes equations. Trudy Mat. Inst. Steklov 125, 196-210 (1973) (in Russian) [English transl.: Proc. Steklov Inst. Math. 125, 186-199 (1973)]

48. Solonnikov, V.A.: On the boundary value problems for systems elliptic in the sense of A. Douglis - L. Nirenberg, I. Izv. Akad. Nauk SSSR Ser. Mat. 28, 665-706 (1964); II. Trudy Mat. Inst. Steklov 92, 233-297 (1966) (in Russian)

49. Solonnikov, V.A.: Solvability of the problem of plane motion of a viscous incompressible capillary liquid in an open container. Preprint LOMI P-5-77, Leningrad (1977) 3-19 (in Russian)

50. Solonnikov, V.A.: Solvability of the problem of plane motion of a heavy viscous incompressible capillary liquid partially filling a container. Izv. Akad. Nauk SSSR 43, 203-236 (1979) (in Russian) [English transl.: Math. USSR-Izv. 14, 193-221 (1980)]

51. Solonnikov, V.A.: On a free boundary problem for the system of Navier-Stokes equations. Trudy Sem. S.L. Sobolev 2, 127-140, 1978 (in Russian)

52. Solonnikov, V.A.: On the Stokes equation in domains with nonsmooth boundaries and on a viscous incompressible flow with a free surface. Nonlinear partial diff. equations and their applications, College de France Seminar 3, 340-423, 1980/1981

53. Solonnikov, V.A. : Solvability of a three-dimensional value problem with a free surface for the stationary Navier-stokes system. Zap. Nauchn. Sem. LOMI 84, 252-285, 1979 (in Russian); see also Partial Differential Equations, Banach Center Publ. 10, 361-403 (1983)

54. Solonnikov, V.A. : Solvability of a problem on the flow of a viscous incompressible liquid into an infinite open basin. Trudy Mat. Inst. Steklov 179, 31, 5 (1987)

55. Solonnikov, V.A.: Estimates of solutions of an initial-boundary value problem for the linear nonstationary Navier-Stokes system. Zap. Nauchn. Sem. LOMI 59, 178-254 (1976) (in Russian)

56. Solonnikov, V.A.: On the solvability of the second initial-boundary problem for the linear nonstationary Navier-Stokes system. Zap. Nauchn. Sem. LOMI 69, 200-218 (1977) (in Russian)

57. Solonnikov, V.A.: Solvability of the problem of the motion of a viscous incompressible fluid bounded by a free surface Izv. Akad. Nauk SSSR Ser. Mat. 41, 1388-1424 (1977) (in Russian) Math. USSR-Izv 11, 1323-1358 (1977)

58. Solonnikov, V.A.: Solvability of the evolution problem for an isolated mass of a viscous incompressible capillary liquid. Zap. Nauchn. Sem. LOMI 140, 179-186 (1984) (in Russian)

59. Solonnikov, V.A.: On an unsteady flow of a finite mass of a liquid bounded by a free surface. Zap. Nauchn. Sem. LOMI 152, 137-157 (1986) (in Russian)

60. Solonnikov, V.A.: On an unsteady motion of an isolated volume of a viscous incompressible fluid. Izv. Akad. Nauk SSSR Ser. Mat. 51, 1065-1087 (1987) (in Russian)

61. Teramoto, Y.: The initial value problem for viscous incompressible flow down an inclined plane. Hiroshima Math. 15, 619-643 (1985)

62. Valli, A.: On the existence of stationary solutions to compressible Navier-Stokes equations. Ann. Inst. Henri Poincaré 4, 99-113 (1987)

63. Valli, A., Zajaczkowski, W.M.: Navier-Stokes equations for compressible fluids: Global existence and qualitative properties of the solutions in the general case. Commun. Math. Phys. 103, 259-296, 1986

Communicated by A. Jaffe

Received June 5, 1989 